The copyright of this thesis vests in the author. No quotation from it or information derived from it is to be published without full acknowledgement of the source. The thesis is to be used for private study or noncommercial research purposes only.

Published by the University of Cape Town (UCT) in terms of the non-exclusive license granted to UCT by the author. 


\title{
Co-Present Photo Sharing on Mobile Devices
}

\author{
Leonard Martin Ah Kun
}

May 2008

Dissertation submitted in full fulfilment for the degree of

Master of Science in Computer Science

Department of Computer Science

University of Cape Town 


\section{Abstract}

This dissertation researches current approaches to photo sharing. We have found that most current methods of photo sharing are not as compelling as traditional photo sharing - with the increasing in popularity of digital photography, consumers do not print photos as often as before and thus typically require a group display (such as a PC) to view their photographs collectively.

This dissertation describes a mobile application that attempts to support traditional photo sharing activities by allowing users to share photos with other co-present users by synchronizing the display on multiple mobile devices. Various floor control policies (software locks that determine when someone can control the displays) were implemented. The behaviour of groups of users was studied to determine how people would use this application for sharing photos and how various floor control policies affect this behaviour. 


\section{Acknowledgements}

Firstly, I would like to thank my supervisor Gary Marsden. He always saw the entertaining side of research and I would like to thank him for all the advice, laughs and motivation he had given me with throughout my studies.

I would also like to thank my friends and family for all their support and inspiration that kept me going throughout my M.Sc.

The handsets for this development work were provided by a donation from HP and bridges.org. This work was funded by South Africa's National Research Federation as well as Telkom and Siemens South Africa through their 'Centres of Excellence' initiative. 


\section{Table of Contents}

Acknowledgements...1. Abstract

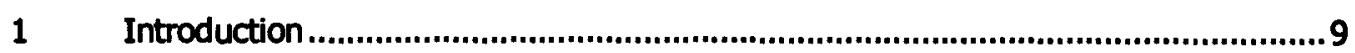

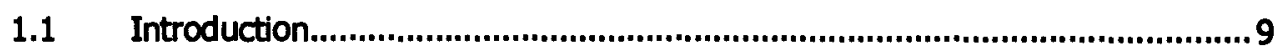

1.2 Digital Cameras and Mobile Devices.................................................... 10

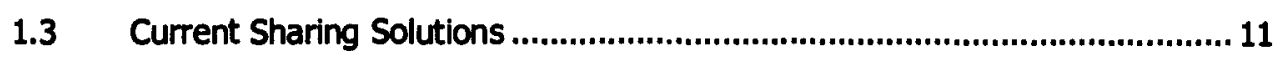

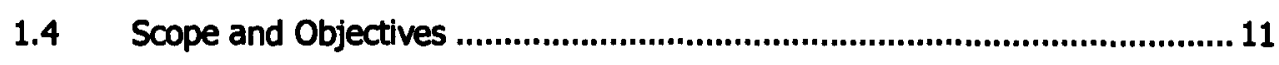

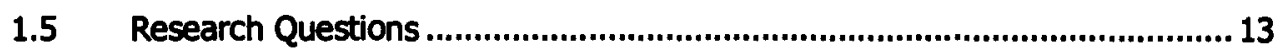

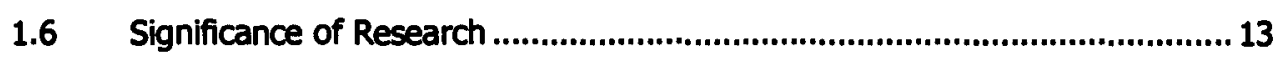

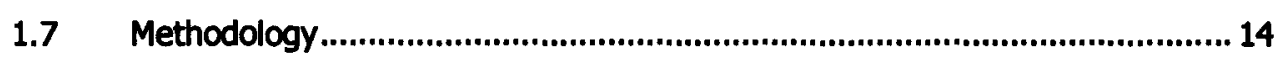

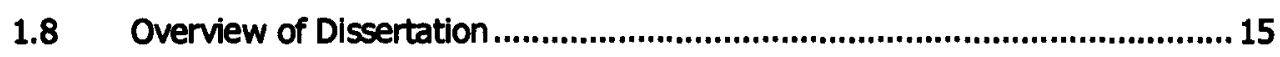

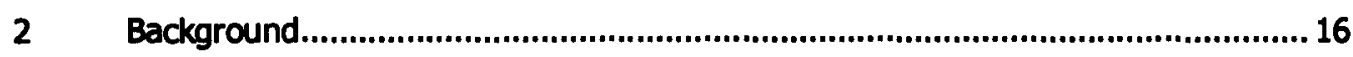

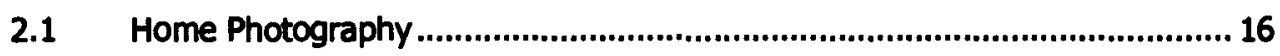

2.2 Conventional Paper-Based Photo Sharing..............................................17

2.2.1 Photo-talk: Storytelling and Reminiscing...........................................17

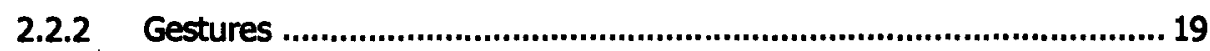

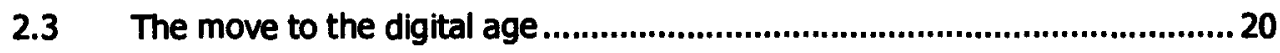

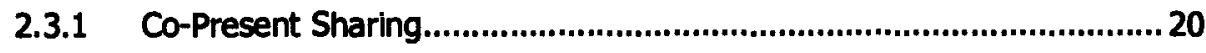

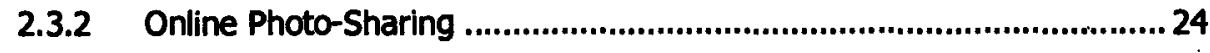

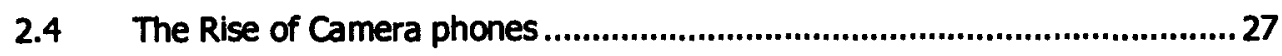

2.4.1 Multimedia Messaging ................................................................... 27 


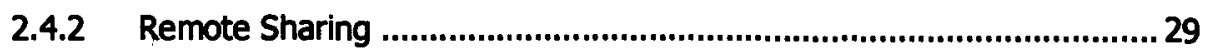

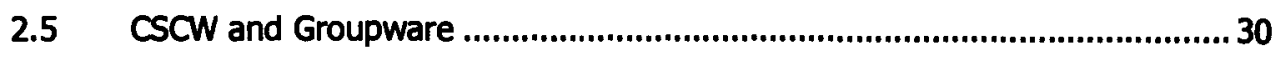

2.5.1 CSCW/Groupware Classification ................................................. 30

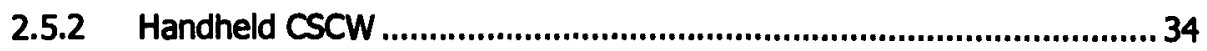

2.5.3 Requirements for Photo Groupware ..................................................... 34

2.6 Summary and Design Implications ....................................................... 36

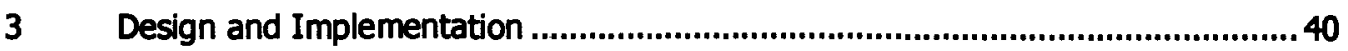

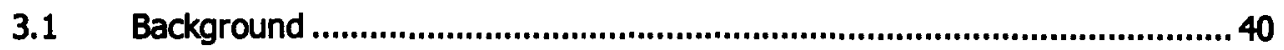

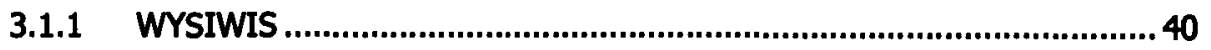

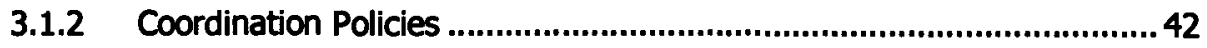

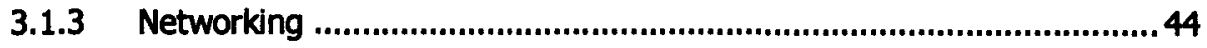

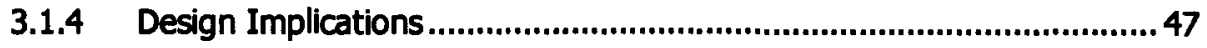

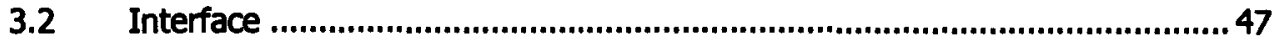

3.2.1 Heuristic evaluation......................................................................... 50

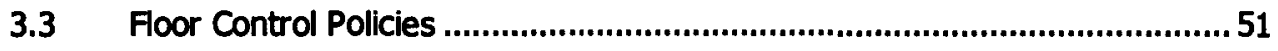

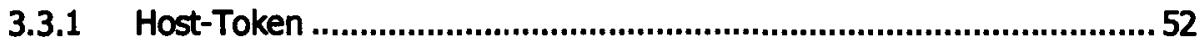

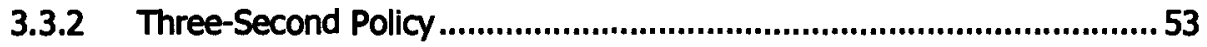

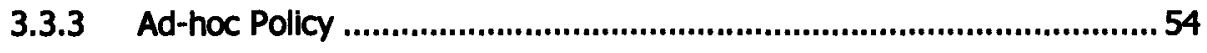

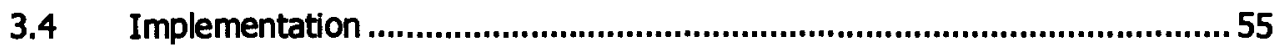

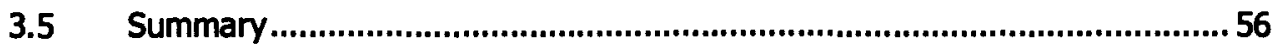

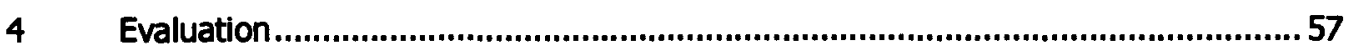

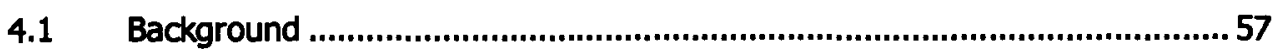

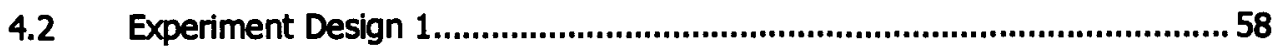

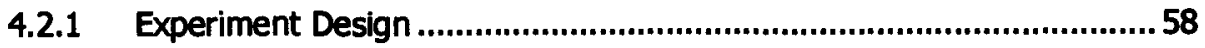

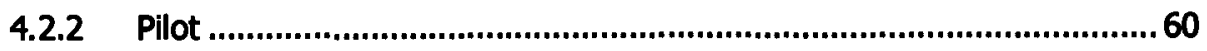

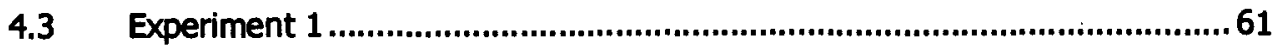




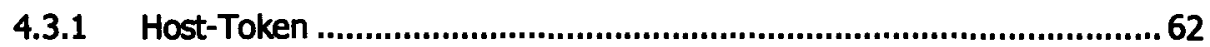

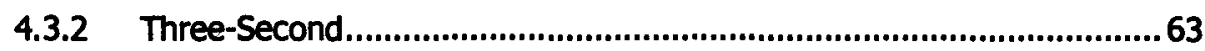

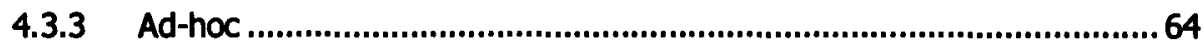

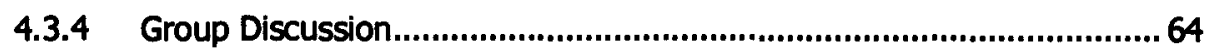

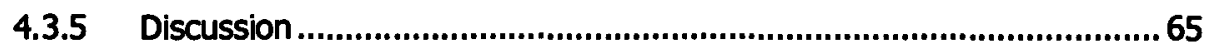

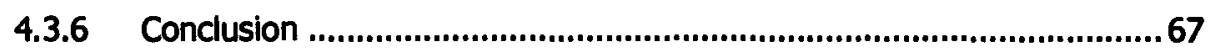

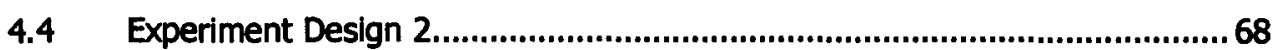

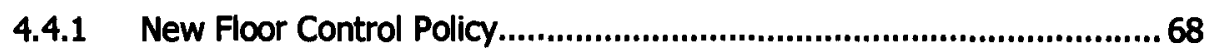

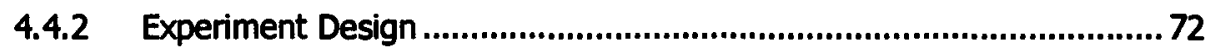

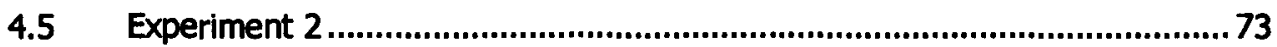

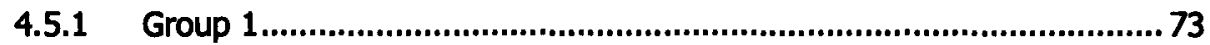

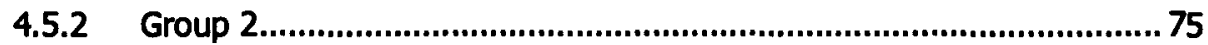

4.5.3 Discussion ............................................................................................ 76

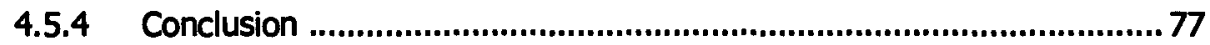

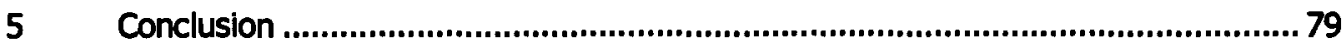

$5.1 \quad$ Research Questions .......................................................................... 79

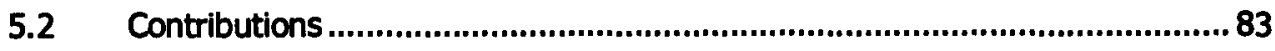

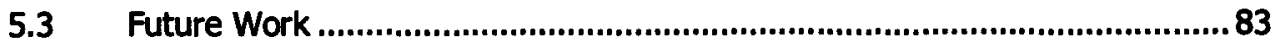

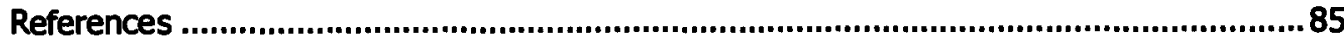

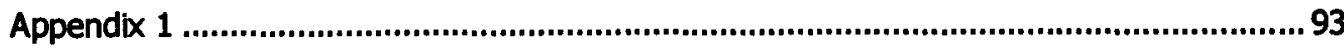

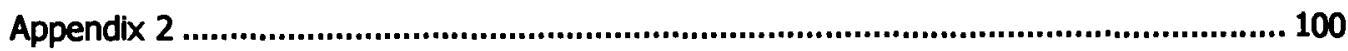

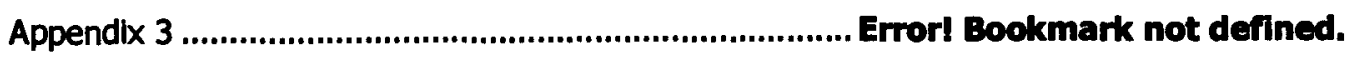




\section{List of Figures}

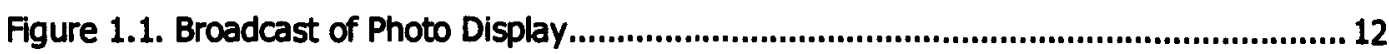

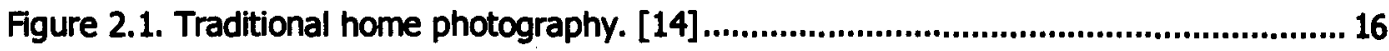

Figure 2.2. The use of pointing to bring attention to a people in photos. [14] .......................19

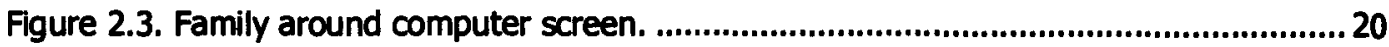

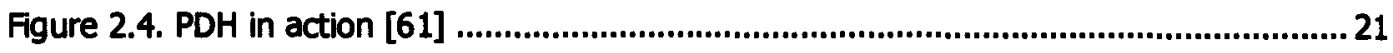

Figure 2.5. StoryTrack in action [4]

Figure 2.6. Sharing with mobile phones. Photo: Fiona-Lee Quimby. ................................... 27

Figure 3.1. iPAQ Image Zone's thumbnail browser ..........................................................48

Figure 3.2. iPAQ Image Zone's image zooming/exploring interface....................................49

Figure 3.3 shows our interface after the heuristic evaluation. Refer to Appendix 1 for a more

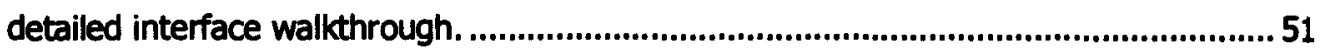

Figure 3.4. Prototype interface after heuristic evaluation.............................................. 51

Figure 3.5. Illustration of host-token request and release. ...............................................52

Figure 3.6. Illustration of Three-Second Floor Control Policy .............................................5 53

Figure 3.7. Ad-hoc Policy, all users can simultaneously send control data...........................54

Figure 4.1. Four friends using the system with the image from one of the PDAs Inset in the top

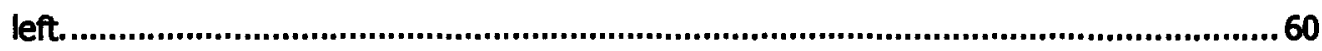

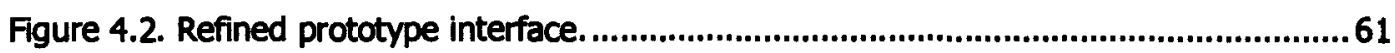

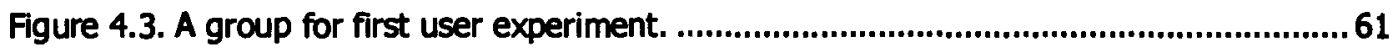

Figure 4.4. Illustration of New Policy.............................................................................. 71

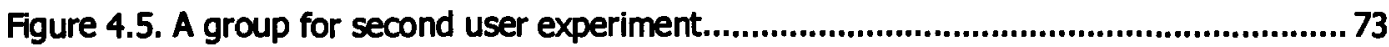




\section{List of Tables}

Table 2.1. A taxonomy of image capture, showing numbers and proportions of images by

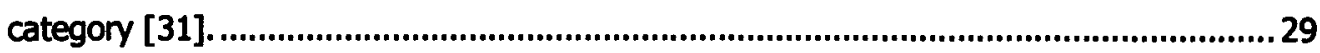

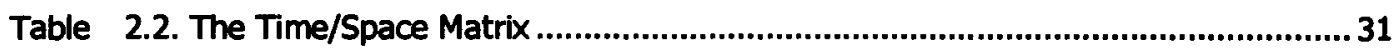

Table 2.3. Categories of photoware with example products and services - adapted from [24] 


\section{Introduction}

\subsection{Introduction}

Photography involves recording images by means of capturing light on a light-sensitive medium or sensor. Traditionally, film was used in conjunction with a camera to record these still moments. The Kodak Brownie was a simple inexpensive camera produced by Kodak. It popularised low-cost photography and was intended to be a camera that anyone could afford and use. This was the start of "home photography", where everyday people could capture memorable moments of their lives rather than just the occasional portraits captured by professionals. Home photography included the anticipation of waiting for the captured photographs to be developed. Once the photos had been developed, they were typically shared with friends or family co-presently, where photos were passed around and stories shared of the experiences captured. Some consumers invest in photo albums to store and organise their photographs and photo frames to display photos in their surroundings. Chalfen [11] called this culture "Kodak Culture" which included the taking and sharing of photographs and video footage of special occasions for the home. This culture involved the social interaction produced by photography whereby people would tell stories and reminisce together, spontaneously triggered by these photographs.

The advancement in technology brought about the development of sensors which allowed photographs to be recorded digitally. The manufacturing costs of these sensors are now low enough for consumer digital cameras to be mass produced and replace traditional film cameras. Digital cameras are now standard for new camera consumers. Cameras are also now integrated in most mobile phones available today. With the increasing popularity of digital cameras and camera phones, many 
consumers are now replacing their traditional film cameras with those that can capture photos in digital format.

Capturing photographs in a digital format has many advantages over film cameras: photographs can be captured at close to no cost; instant review of a captured photo is possible; the medium (memory card) used to store these photos can be reused; many more photographs can be stored on these memory cards compared to film and these photographs are extremely portable and can be duplicated and shared almost instantly at little or no cost. The disadvantage of digital cameras, however, is the decrease in consumers printing their digital photos; and thus the social experience of sharing photos in the traditional way has decreased commensurately.

\subsection{Digital Cameras and Mobile Devices}

Digital cameras are increasingly becoming standard for capturing photos [47]. Many mobile devices and mobile phones are now equipped with colour screens that can be used to display photos. These displays are typically small and are only really designed for personal viewing. In addition to music, the iPod which is the most popular mobile music player in the world [56] now allows you to store and display your photo collection.

Most mobile phones now come with an integrated camera and high resolution screens. The quality of these cameras is constantly improving and manufacturers are now selling camera phones as digital cameras (e.g. Sony Ericsson Cyber-shot phones). These camera phones are starting to be used as personal flipbooks to view photos captured by consumers [31]. 


\subsection{Current Sharing Solutions}

For digital images captured by cameras and camera phones, there are many options for sharing these images. The desktop computer screen is typically used to show photos to a larger group when in a social gathering; however, this is inconvenient as the group is required to go to the screen to view the photos rather than bringing the photos to the group. Many cameras can also be connected to a television set and photos can be viewed via the large TV screen. These extemal displays are usually inconvenient when wanting to share photos outside the home. Research on co-located photo sharing solutions include tabletop displays [1] and Balabanovićs StoryTrack [4]. Tabletop displays allow for more social interactivity when sharing, however, does not address the problem of portability. Balabanovic's StoryTrack makes use of a tablet PC, however it is limited to sharing photos with only a small group of people.

Other photo sharing research and services for photo sharing are targeted at remote sharing. Typical remote photo sharing includes using a desktop computer to send photos to others via e-mail and upload images to websites such as flickr, myspace, facebook, etc. and other photo sharing/blog type websites. Photos captured with camera phones can be shared with other mobile phones by transferring them via infra-red, Bluetooth or MMS. Photos can also be posted on the intemet via these devices to various mobile photo sharing websites and mobile-blogs. These services are great for sharing photos with remote people, but do not support the enjoyable social activity of co-present photo sharing.

\subsection{Scope and Objectives}

With these new digital cameras and camera phones, consumers are capturing more photos and sharing them more frequently. Using websites to share photos are great 
solutsons provided you are not intending to share images with multiple co-present people. We know from studies such as Kindberg [31] and Frohlich [24] that co-present simultaneous sharing is a desirable social activity. To achieve this with current cameras and camera phones one must connect the device to some external display. This is, at best. inconvenient when meeting with friends in a home, pub or restaurant. Most people now possess a mobile phone which essentially includes a personal display. We were therefore interested in pursuing an approach whereby any user in a group couid broadcast an image onto the screens of other mobile devices that belong to other people in that group.

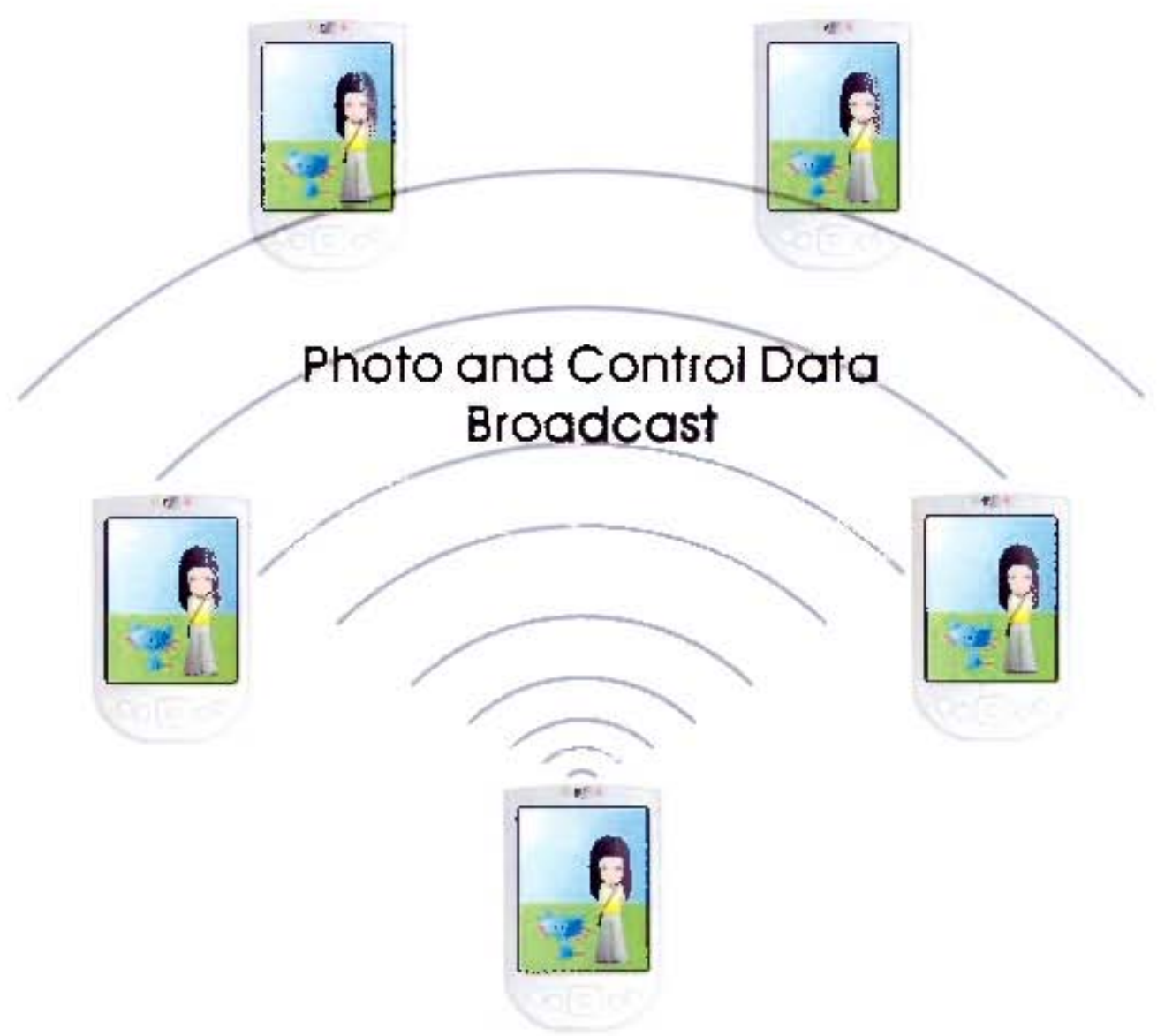

Гigure 1.: Brcatcast of Fhntr .jisplay 


\subsection{Research Questions}

Two main questions drove our research design:

- Would people really be interested in seeing someone else's photos on a mobile device - or will the screen simply be too small to be engaging? We wanted to determine whether users would find sharing photos in this way compelling and usable and whether there is a viable solution to co-present photo sharing with digital photographs. Would the natural interaction such as storytelling and reminiscing that occurs with traditional photo sharing still occur with our system? How does our system compare to sharing photos in the traditional way? What other behaviours might our system produce?

- How should the system manage the social interaction - should anyone be allowed to broadcast an image at any time, or does control need to be moderated through some convention such as token passing? Since it is possible for all users to interact with the displayed photos, we wanted to determine if users could coordinate themselves without including any strict policies. Since this system will be used for social purposes, it is important to understand the possible conflicts and how users can interact with each other.

\subsection{Significance of Research}

The need for photo sharing software that supports engaging and compelling experiences have been identified by numerous researcher papers [24][4][43][14]. Most photo sharing software available today does not target simultaneous co-present sharing. Sharing photos in this manner is a desirable activity [24][14] and thus our 
research will pursue a new interaction technique to support co-present sharing. The prototype developed as part of this work is intended to promote co-present sharing that supports, and possibly improves on, the social activities found in the traditional method of sharing photos.

\subsection{Methodology}

This research will be based on human-centred design techniques, specifically Interaction Design. Preece et al. describe interaction design as "designing interactive products to support people in their everyday lives" [51]. Jones and Marsden [35] describe three main types of activities involved in effective interaction design.

- Understanding users

- Developing prototype designs

- Evaluation

Understanding users involves having a sense of people's capabilities and limitations; gaining a rich picture of what makes up the detail of their lives, the things they do and use. To understand our users, background research on traditional and current photo sharing practices will be conducted. This will help us understand how and why people share photographs and how the advancement of technology affects that.

Developing prototype designs involves representing a proposed interaction design in such a way that it can be demonstrated, altered and discussed. For our prototype, we research and discuss various issues involving the technical side of implementing our system.

For the evaluation of our prototype, we review various methods to evaluate our system and propose our experiment design. Initial evaluations include heuristic 
evaluations and pilot study. Thereafter our experiment will be conducted. With our findings, our prototype will be refined and another experiment conducted.

\subsection{Overview of Dissertation}

The following chapters follow that of the interaction design methodology used.

- Chapter 2 describes our background research to understand how users share photographs traditionally and in our current digital age.

- Chapter 3 describes the design of our prototype and various floor control policies that manage the interaction between users.

- Chapter 4 describes our evaluation of our prototype. It also includes the details of our second refined refinement prototype and evaluation.

- Chapter 5 describes our major findings and relates them to our research questions. Possible future work is also described here. 


\section{Background}

\subsection{Home Photography}

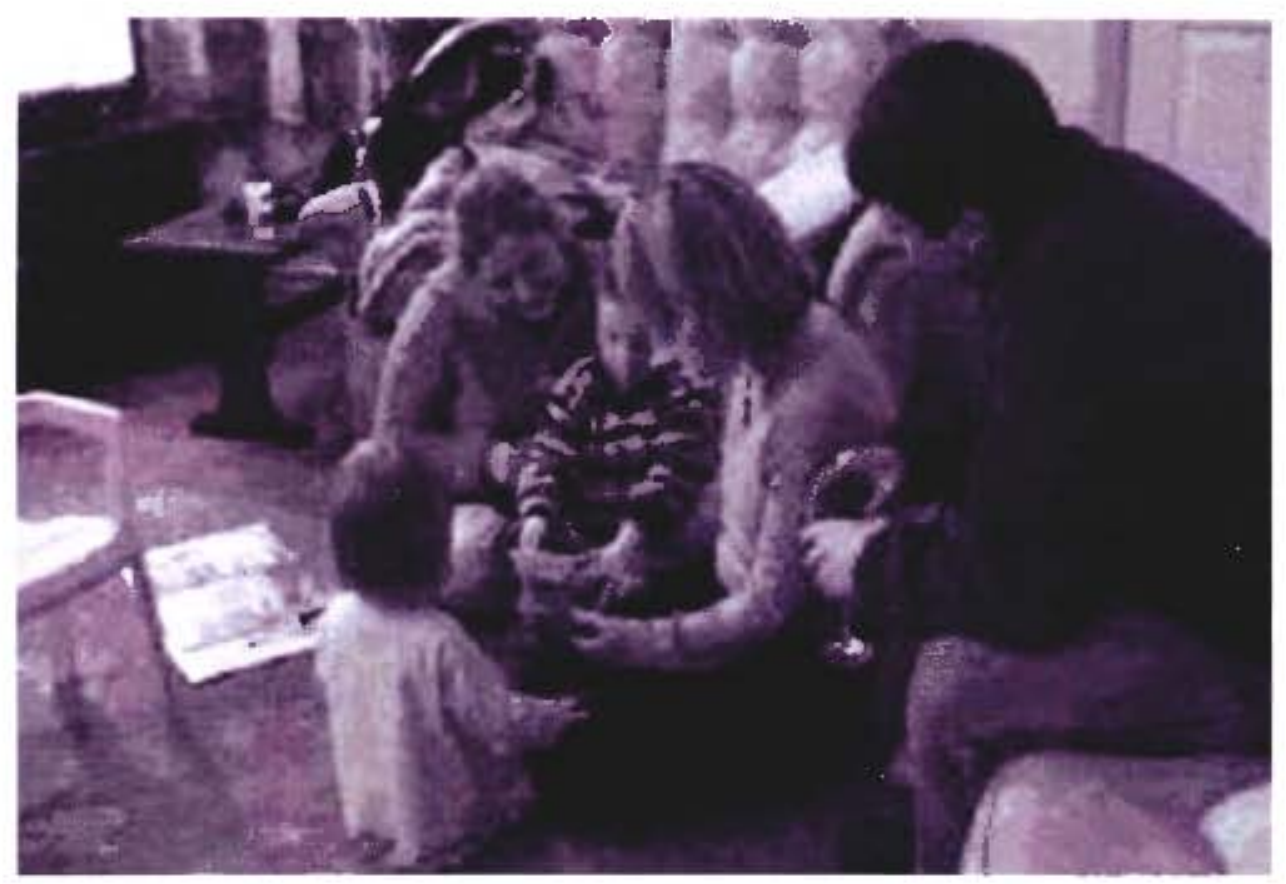

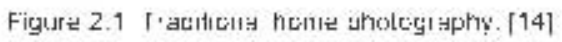

Communication of experience" is the main reason for sharing photos [11]. One of the most common and enjoyable tses for photographs is to share stories about experiences, travels, friends and family. This is what anthropologist Richard Chalfen found whie studying the functions of home photography. He identified that home photography is a particular type of practice that is concerned with the creation and Ltilization of pictorial artefacts in the context of the home These artefacts include snapshots. family albums, slides and home videos.

Chalfen describes five examples of photograph tse [38].

- Shared photographs - peopie like to share photos with the family, friencis or colleagres. 
- Household photography - photographs within the home (on walls, furniture, etc).

- Work photography - snapshots that are taken and displayed at work.

- Wallet photography - family snapshots kept in wallet.

- Tourist photography - photographs taken when travelling.

From these practices, it was revealed that photographs act as social artefacts that can trigger affective processes [38]. Photographs often recall memories that emotionally affect the viewer, provoking emotions such as happiness, sadness or anger. This suggests that sharing photographs is a social process that connects people together.

Koskinen et al. [33] also identified photos being used as a tool for remembering. They point out that remembering consists of stories which create closeness within a small circle of people and that browsing photographs is a group activity which brings about the feeling of connectedness among participants.

\subsection{Conventional Paper-Based Photo Sharing}

\subsubsection{Photo-talk: Storytelling and Reminiscing}

The term 'Kodak Culture' was defined by Richard Chalfen [11], who identified this group of people as those who typically share photographs or video footage of traditional subjects such as celebrations and family holidays. Those in the Kodak Culture enjoy engaging in photographs and telling stories about them. They share oral stories around photographs with others who can share and build on their narratives. Frohlich called this naturally occurring conversation 'photo-talk'.

Frohlich and his colleagues studied conventional photo-sharing practices. They established that people organise their photos in albums for social purposes or events 
[24]. They identified two categories of photo-talk. Storytelling photo-talk occurred in conversations where photos were shown to others who were not there at the time the photos were captured and hence did not share the memory represented by the photos. The value of storytelling was found in communicating status, experience and wisdom to others. They also found that in situations where more than one person who shares the memory represented in the photo is present, this storytelling often becomes a collaborative project. Reminiscing photo-talk occurred in conversations when sharing photos exclusively with members of the original "capture group". The value of reminiscing is found in the recalling of the details of the experiences with others.

Crabtree et al. conducted an ethnographic study on 22 families in the UK to investigate the collaborative use of paper-based photos in the home environment [14]. The researchers found that photo-talk is an "achieved feature of collaboration" where the use of photographs is usually coordinated by the participants from some central point (table, sofa, floor, etc). This central point acts as a mutually accessible region where people can gather around and view photographs.

It was observed that photographs do not only act as conversational resources for the holder of the photographs, but are also conversational resources for the other participants as well. The participants may ask questions upon viewing a certain photograph of the holder and raise conversational topics that may result in the telling of a story. This behaviour was also observed by Frohlich who found that not all stories relate to the photographs at hand. It is thus noted that having a flexible group view of photographs is important where the collaborative use of photographs is concerned.

Crabtree's findings echoed Frohlich's findings that sharing photos exclusively with members of the original 'capture group' resulted in reminiscing talk. This involved the 
recalling of details of the experiences, jointly 'finding' the memory together, only to leave the memory unelaborated. It was rare to find members of the original capture group to re-tell stories that they have experienced together. This shows a mutual understanding or common ground in conversation.

CrabTree et al. identified three essential components for accomplishing photo-talk

1. The organization of photos for ready retrieval

2. The manipulation of the collection

3. The production of an account that gives the photos in hand their particular meaning

The nature of storytelling is based on 'recipient design' which was identified by a number of researchers such as Chalfen and Frohlich. This meant that stories were told differently depending on to whom the story was being told. These conversations display an orientation and sensitivity to specific co-participants.

\subsubsection{Gestures}

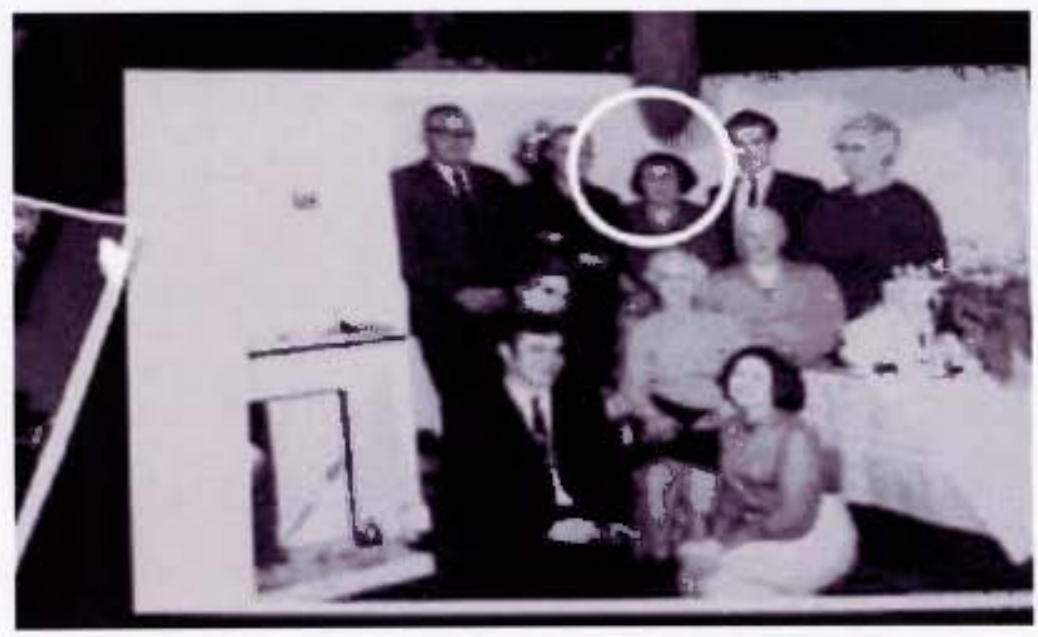

Figure 2.2. The use of potnting to bring artention to a people in photos. [14] 
Frohlich [24] and Balabanovic [4] noted that participants want to point at things when talking about photos. Crabtree and his collaagues discuss these gestural practices (i.e. pointing: tapping, etc) and their importance to the production of accounts. They determined that these gestures highlight objects for perception and draws attention to what is being talked about. It also gives photographs their sense of reference or meaning while sharing photos with co-present others.

\subsection{The move to the digital age}

Initially industry focused on facilitating home development of photos [24]. This included the development of digital cameras, photo scanners and photo-quality printers which essentially emulated conventional photography in the home environment. Support for storage. sending and sharing photos online later started to emerge.

\subsubsection{Co-Present Sharing}

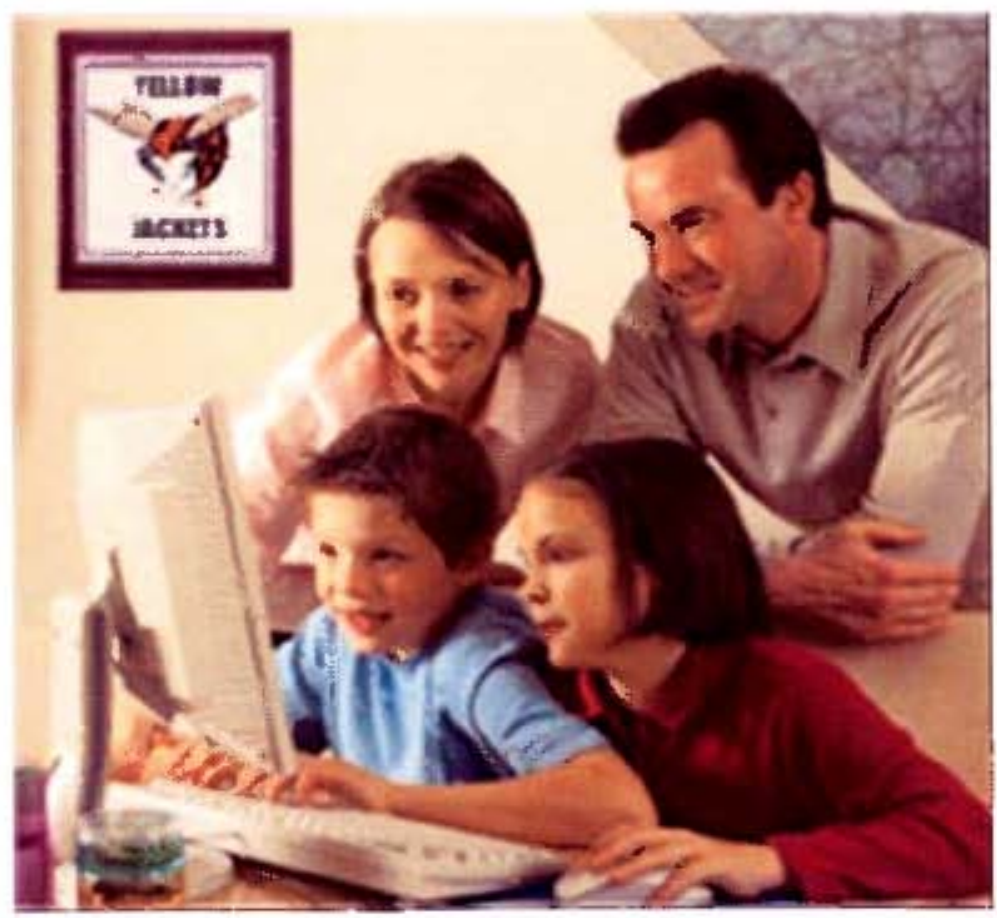

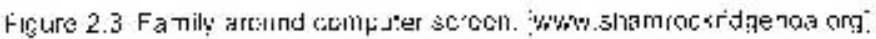


Digital photographs are generally viewed on personal computer ( $\mathrm{PC}$ ) screens as this is typically where digital photos are downloaded. stored and managed. These photographs are viewed via image viewers or image database products such as ACDSee [2]. Picassa [49], IPhoto [28] and Adobe Photoshop Album [3]. These applications were developed to be used on a single desktop PC which typically does not facilitate shared interaction (i.e. typically only one person is in control of the PC while others just watch), thus other approaches have been pursued.

\subsection{1:1 Tabletop interfaces}

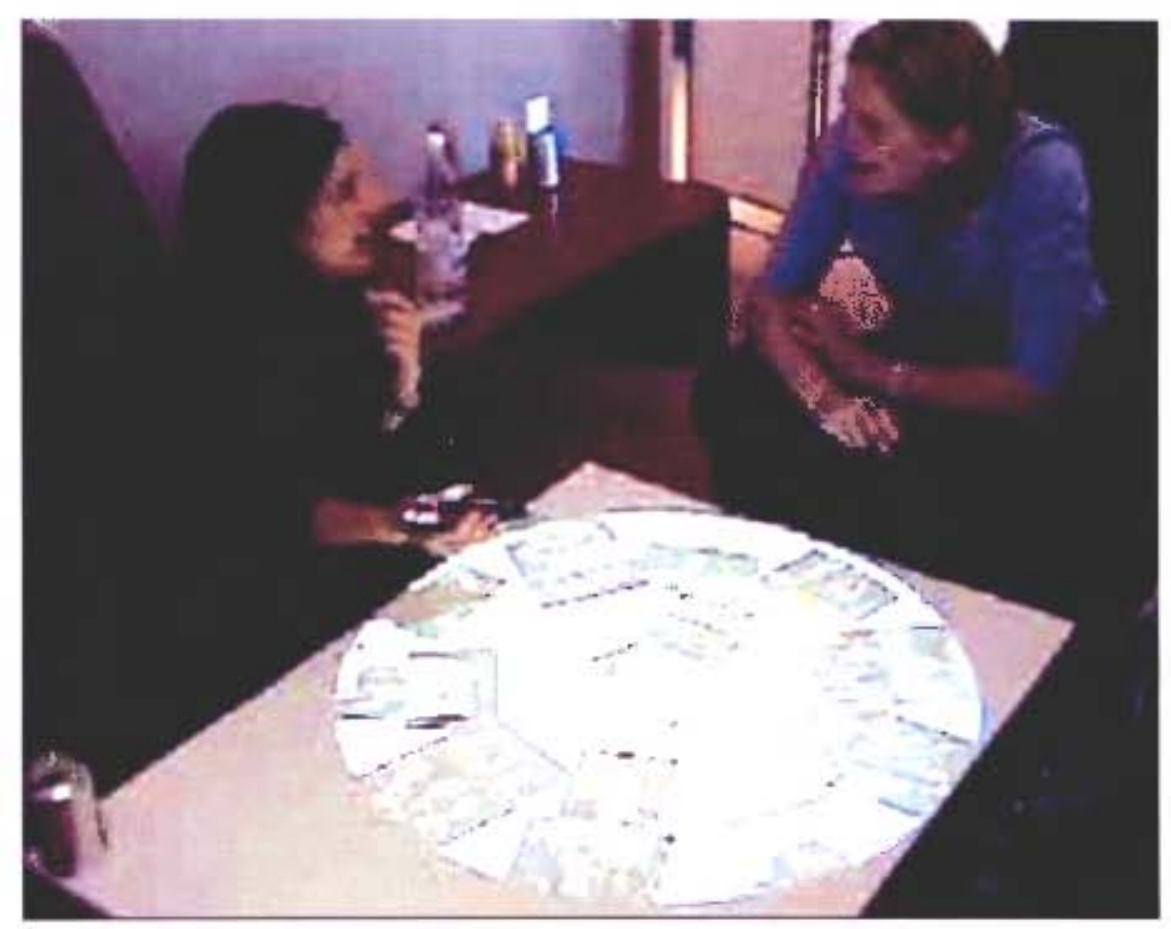

Figure 2.4 P.211 n action [bil]

Shen et al. presented a photo viewer called the photo digital historian that supports conversational multi-person viewing wit. an emphasis on storytelling [61]. Their research showed that tabletop displays are ideal for collaborative tasks as people can sit around the table and all face each other, rather than crowd around a desktop or small handheld device. It is suggested that the traditional interface is not well suited to 
support collaboration because it forces people to face in the direction of the screen. This design assumes that people will always be viewing objects from roughly the same direction and angle. Recognition of this importance of physical orientation has led to the development of circular tabletop interfaces that enable users to push photos around the table and orient them to one another [63]

Apted et al. explored photo sharing for the elderiy using the tabletop interface [1]. The research focused on here was on design guidetines to make tabletop interfaces highly learnable and usable for both young and old users.

\subsubsection{Story Track}

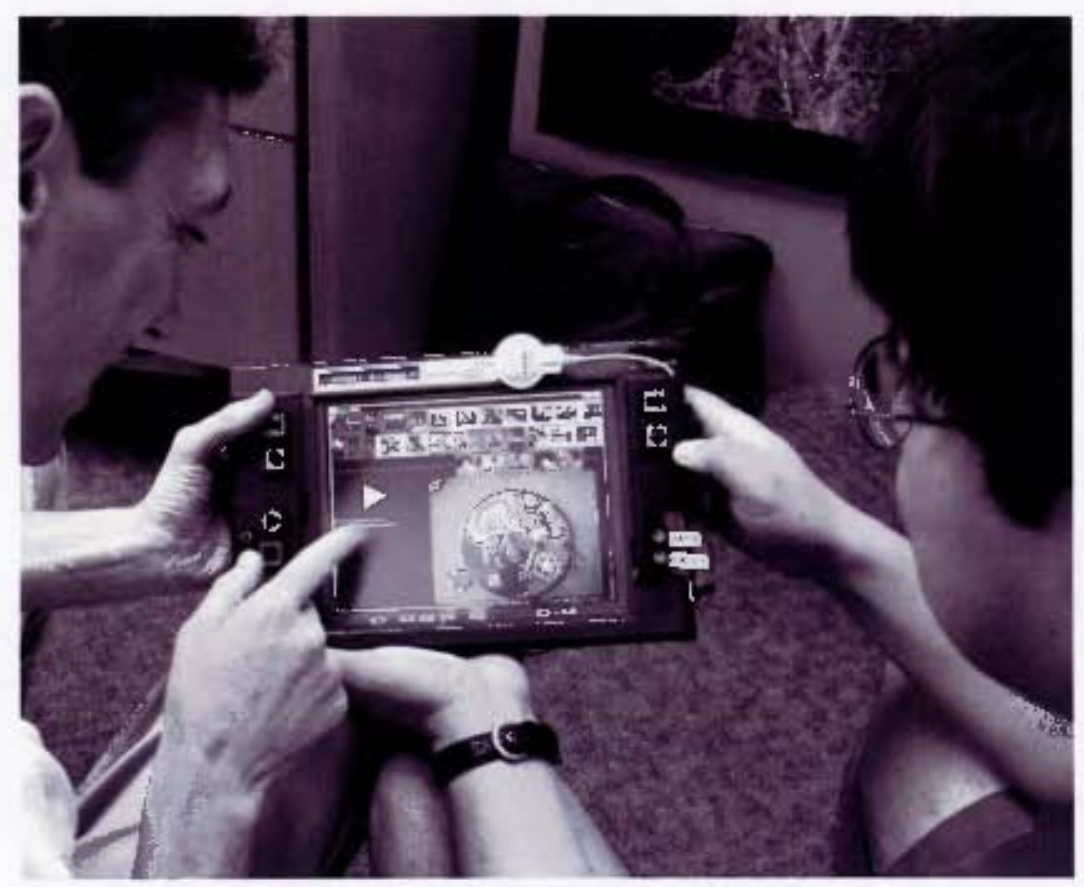

Higure 2.5. Story Track in action [4]

A prototype device called StoryTrack that enables digital photos to be used in a manner similar to print photos for sharing personal stories was developed [4]. The prototype addressed two different modes of interaction. 
1. Sharing of stories locally, that is, with at least one other person present and viewing the same photos;

2. Sharing of stories remotely, that is, by sending someone a set of photos along with some commentary

This was achieved with a mobile device that could be passed around like a regular photo album to view digital photographs. Balabanovic et al. state that sharing photos in a natural setting requires a portable device that can be used in different locations throughout one's home. The devices should also be large enough to show photos at a size similar to regular prints viewable by more than one person. It is also mentioned that control should be easily passed from one user to another. The device used for their prototype was a tablet PC. Balabanovic et al. avoided using a touchscreen or pointing device as input as people point at pictures when talking about them and using the same gestures to control the device might be confusing and produce unexpected behaviour.

From their observations of their evaluation, Balabanovic et al. identified two different styles of storytelling.

1. Photo-driven: this is where a subject explains every photo in turn, the story prompted by the existing sequence of pictures. This type of storytelling was typically found when sharing photos with remote users.

2. Story-driven: the subject has a particular story in mind, then gathers the appropriate photos and recounts the story. This type of storytelling was typically found when sharing with co-present users.

The conclusion of this paper is that this device demonstrates that digital photos can be used to support some of the same kinds of sharing practices that people enjoy with 
print photos. Participants could pass the device around to others and also physically rotate the device if desired. One complaint however was that the device was found to be too heavy for most users.

\subsubsection{Online Photo-Sharing}

Online photo-sharing initially included practices such as sending photos via e-mail and posting photos on websites. Miller et al. examined digital photographers' photo practices [43]. Their research focused on the online photo-sharing solution, Flickr.com [21]. Flickr.com is a website that allows users to store, search, sort and share their photos online.

They identify two distinct classes of users, the digitally adapted 'Kodak Culture' and another group that they have termed 'Snaprs'. Miller et al. acknowledged that Kodak Culture practices still takes place with digital photographs and wanted to determine whether sites like Flickr.com support these practices. The term 'Snaprs' was given to the group of users because of their common membership in a Flickr group and their unique practices which were bound to Flickr-specific capabilities.

Snaprs were found to be more of the hobbyist photographers, who upload photos to Flickr and give comments and critique to their fellow Snaprs' photos. Snaprs often took photos at least once a week. The reason for Snaprs taking photos was centred around the taking of pictures, not the sharing of them. Snaprs typically upload their photos to a website such as Flickr, to share with a community of people they do not know personally. The photos taken are more of an artistic nature rather than personal photos and are focused on improving their photo-capturing technique. Kodak culture participants on the other hand took far fewer pictures (once a month or several times a year). The photos taken were typically of holidays or trips to archive these events and share with their social network of family and friends. The primary method of 
sharing remotely for Kodak Culture people were via e-mail while snappers preferred sharing their photos via the Flickr website.

Miller and his colleagues found that Flickr type websites enable global photo-sharing between strangers and encourages the formation of online communities around different photographic styles or subjects. This works well for Snaprs, but not for Kodak Culture photographers. Kodak Culture photographers wanted to share stories with existing family and friends and were found to prefer offline sharing practices and printing their photos. E-mail is the method closest to supporting remote sharing for Kodak Culture people. An e-mail message is intentional, in that photos are actively shared rather than simply added to an online folder.

Vronay et al.'s research investigated why sharing digital photographs on the internet is not as compelling as sharing photographs face-to-face [64]. They found that this was related to the importance of emotion in the computing experience. Importance of emotion in photo-sharing practices was also identified by Koskinen [33]. Photographs trigger memories, which trigger emotion. Photos are about people and their stories which are not supported when sharing photos on the internet. The following are problems Vronay identified related to internet photo-sharing websites:

- The uploading of photos to the website is tedious

- There is no easy way to attach voice/text annotations.

- You cannot highlight or point at items on photos.

- Annotations are typically generic and less emotional as you cannot provide a different annotation for different people.

- When browsing through photos yourself, there is no information as to which photos are most interesting. 
Vronay and his colleagues designed and implemented an application called PhotoStory to annotate and cinematize (adding motion and sound) a set of photographs. This included voice annotation as speaking while sharing photos is a natural and socially expected noted by Chalfen. They also included the ability to zoom on various sections of the photos that the annotation was referring to which acted as an analogue to gestures. The software focused on narration, emphasis and visual effects. The application produced a video file which could then be sent to others via e-mail. Their results conclude that this approach is more compelling than the standard approach of using still photos. Motion and voice was found to work best when used together (rather than static photos and text). The only disadvantage noted was that some of the emotional warmth and spontaneity is lost. 


\subsection{The Rise of Camera phones}

\subsubsection{Multimedia Messaging}

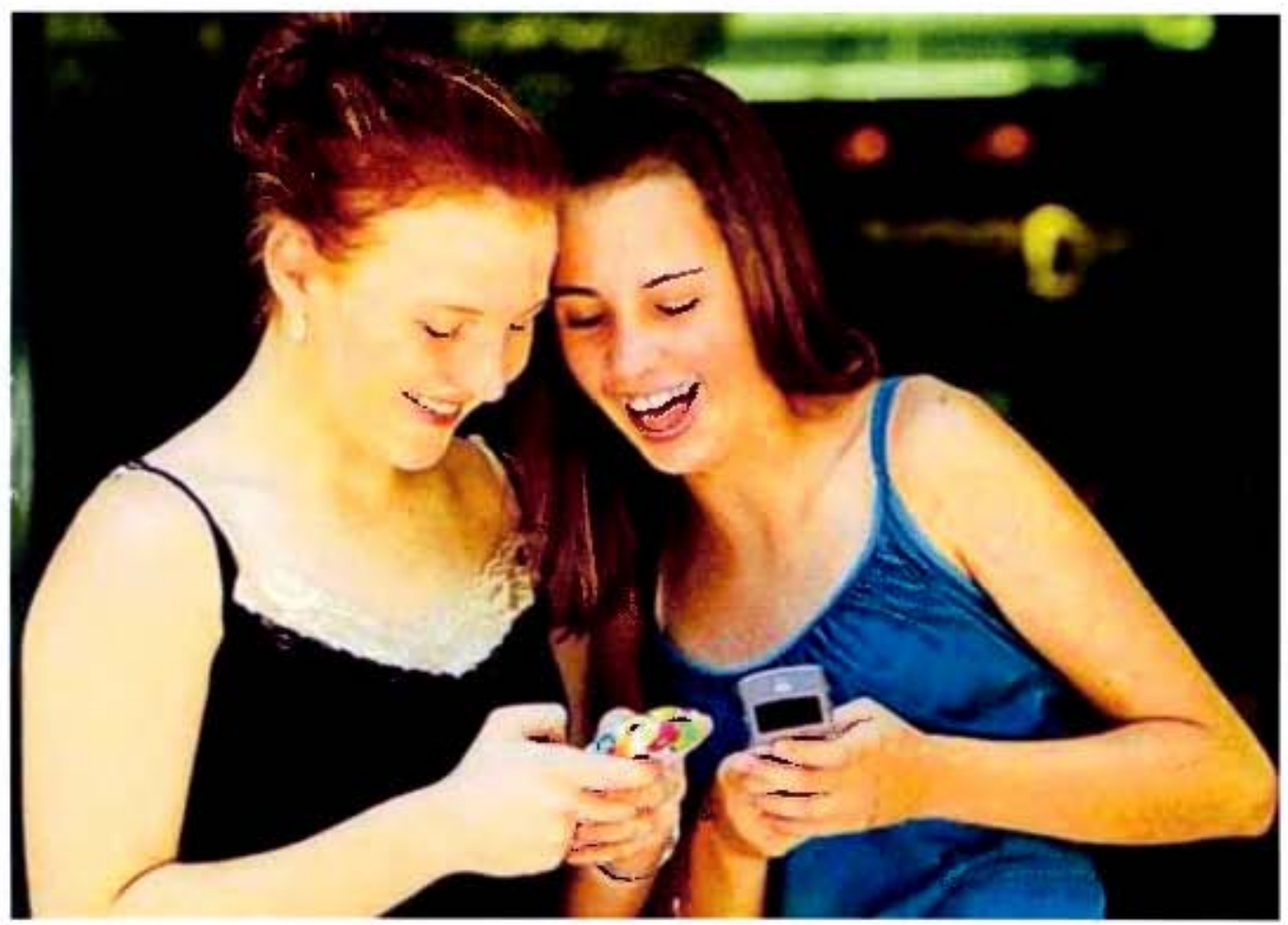

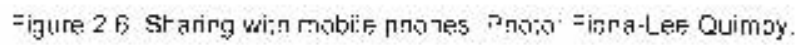

Makkelä et al, ran a field study with a prototype mobile device that sent images to other participants wirelessly [39]. This was pror to the release of commercial camera phones and was part of a product concept design done in Maypole. The Maypole Project was a two year Project with the European Initiative on Intelligent Information Interfaces The research initially focused on children and their social behaviours [12][25]. These observations would be used to inspire new ideas about using communication technology. This prototype allowed participants to capture and save digital images, edit images by adding transitions to a set of images. add sound effects and send their creation to other participants. They found that this type of image sharing supports socialization but does not support practices from conventional 
photo-sharing. The practices found during this field study were of photo-creating, creating stories, art and expressing affection echoing Balabanović's photo-driven practices. This result whereby photos were used to tell stories with images, rather than about images was further supported by Kurvinen's research [34], who studied how people use multimedia messaging with their mobile phones.

Koskinen et al. found similar results. They describe a study in which several groups of users were given digital cameras and mobile phones capable of sending images over a wireless network [33]. Their aim was to determine how real people sent mobile images. They found that images were sent several times, but the text that frames it and gives it a plot is changed for different recipients (recipient design). Images were captured to create stories for jokes and teasing (Balabanovic's photo-driven practices). They commented that there is a lot of interest in technology when people can use it to entertain people.

Kindberg et al. conducted an in-depth study to determine how and why people use their cameraphones [31]. The result was a 6 part taxonomy describing the way images are used for personal, affective and functional use.

\begin{tabular}{|c|c|c|c|c|c|c|}
\hline & \multicolumn{4}{|c|}{ Social } & \multicolumn{2}{|c|}{ Individual } \\
\hline Affective & $\begin{array}{l}\text { Mutual } \\
\text { Experience. } \\
\text { Images used to } \\
\text { enrich a shared, } \\
\text { co-present } \\
\text { experience } \\
\text { (either in the } \\
\text { moment or later } \\
\text { as a momento) }\end{array}$ & $\begin{array}{l}103 \\
(35 \%)\end{array}$ & $\begin{array}{l}\text { Absent Friends } \\
\text { or Family. } \\
\text { Images used to } \\
\text { communicate } \\
\text { with absent } \\
\text { friends or } \\
\text { family (either in } \\
\text { the moment or } \\
\text { later). }\end{array}$ & $\begin{array}{l}63 \\
(21 \%)\end{array}$ & $\begin{array}{l}\text { Personal } \\
\text { Reflection. } \\
\text { Images used } \\
\text { for personal } \\
\text { reflection or } \\
\text { reminiscing. }\end{array}$ & $\begin{array}{l}120 \\
(41 \%)\end{array}$ \\
\hline Functional & $\begin{array}{l}\text { Mutual Task. } \\
\text { Images shared } \\
\text { with people } \\
\text { co-present in }\end{array}$ & $\begin{array}{l}11 \\
(4 \%)\end{array}$ & $\begin{array}{l}\text { Remote Task. } \\
\text { Images used to } \\
\text { help } \\
\text { accomplish a }\end{array}$ & $\begin{array}{l}23 \\
(8 \%)\end{array}$ & $\begin{array}{l}\text { Personal Task. } \\
\text { Images used to } \\
\text { support some } \\
\text { future task not }\end{array}$ & $\begin{array}{l}29 \\
(10 \%)\end{array}$ \\
\hline
\end{tabular}




\begin{tabular}{|l|l|l|l|l|l|}
\hline $\begin{array}{l}\text { support of a task } \\
\text { (either in the } \\
\text { moment or after } \\
\text { the event). }\end{array}$ & $\begin{array}{l}\text { task by sharing } \\
\text { with remote } \\
\text { family, friends } \\
\text { or colleagues } \\
\text { (either in the } \\
\text { moment or } \\
\text { later). }\end{array}$ & & $\begin{array}{l}\text { involving } \\
\text { sharing }\end{array}$ & \\
\hline
\end{tabular}

Table 2.1. A taxonomy of image capture, showing numbers and proportions of images by category [31].

Images found on subjects' phones were mostly photos that subjects had captured themselves rather than received from other people. The participants reported using their cameraphones as personal flipbooks of images. When it came to sharing, most image-sharing took place face-to-face, almost always on the phone's screen but occasionally by direct phone-to-phone transfers or MMS. This behaviour was further re-enforced by Van House [62], Solovaara [55] and Ballagas [5]. Photos captured which were initially not intended to be shared were sometimes still shown to others on the phone screen. Sharing photos face-to-face was described as casual and spontaneous in that sometimes it meant passing the phone to someone else or swapping phones with a friend.

When it came to printing, only 12 of their 34 subjects reported printing photos captured or received on their phone. The most common social reason reported for capturing an image was to enrich a mutual experience by sharing an image with those who were co-present at the time. Kindberg established that the reason why sending photos was not more frequent was due to the expense, complexity and poor image quality.

\subsubsection{Remote Sharing}

With the increasing popularity of camera phones, a number of online services have emerged to support mobile photo-sharing. These services included uploading photos 
to mobile blogs and photo-sharing websites. There are numerous studies based on remote sharing on mobile devices such as MobShare[13], Flipper [59], Mgroup [55] as well as commercial products such as Pictavision [50]. These services are intended to offer visual sharing which represent a type of ambient virtual co-presence/telepresence[31] whereby others know what a particular person is/has been doing by viewing the photos being posted.

MobShare and Flipper took the photoblogging approach. Photoblogging systems provide a way to publish mobile pictures on the web and allow visitors to comment on the published pictures. MobShare included the ability to form new viewer groups and picture albums on the fly. Flipper attempted to automate the photoblogging process by automatically sharing pictures with fixed predefined "buddies" and organising the photos according to who the sender was. This automated sharing requires the user to decide what not to share rather than what to share. Mgroup differs slightly to MobShare and Flipper in that it supports the collective creation of media stories that is created and shared with a group of friends.

\subsection{CSCW and Groupware}

\subsubsection{CsCW/Groupware Classification}

This research might be broadly classified under the area of "Computer Supported Collaborative Work" (CSCW). Fouss and Chang [23] identify the ambiguities of CSCW and groupware classifications. CSCW deals with issues involved in group work and how technology can support the process. This term has been debated as an inappropriate title for the research field. Fouss and Chang mention that the typical complaint is that the words themselves are too restrictive and that strict adherence to 
the actual meaning of the phrase does not encompass the whole area of research that has come under the umbrella term CSCW.

A better term to describe our work is "Groupware" which has also been scrutinized, but less so than CSCW. A general accepted definition for groupware would be any application that in someway supports group activities. The problem identified with this definition is that it is too broad and encompasses many types of applications such as network operating systems and e-mail.

The most common characteristics used to classify groupware are those found in the time/space matrix.

\begin{tabular}{|c|c|c|}
\hline & $\begin{array}{c}\text { Same Place } \\
\text { (Co-Present) }\end{array}$ & $\begin{array}{c}\text { Different Place } \\
\text { (Remote) }\end{array}$ \\
\hline $\begin{array}{c}\text { Same Time } \\
\text { (Synchronous) }\end{array}$ & Face-to-Face Conversation & Telephone \\
\hline $\begin{array}{c}\text { Different Time } \\
\text { (Asynchronous) }\end{array}$ & Post-it note & Letter \\
\hline
\end{tabular}

Table 2.2. The Time/Space Matrix

Synchronous groupware assists a group of individuals in working together at the same time. Asynchronous groupware supports communication and problem solving among groups of individuals who can contribute at different times. Same place means that individuals are in the same location, e.g. conference room. Different place means that individuals are either in different geographical locations or simply not in the same room.

Another characteristic Fouss and Chang use to classify groupware is based on the method used to coordinate activity. Groupware coordination can be sequential, parallel or reciprocal. Sequential groupware only allows one member to be actively working on the project at any given time. The active member can pass the control to another member, who can then work on the project. Parallel groupware allows 
members to work independently on a part of the project simultaneously. With reciprocal groupware multiple group members are working together on a part of the project.

Fouss and Chang define a groupware taxonomy by describing groupware applications and the functionality they provide. Their taxonomy include Messaging Systems, Coordination Systems, Conferencing Systems, Group Decision Support Systems, Multi-User Editors and Collaborative Programming. The groupware that seems to have the most potential in supporting storytelling is that of conferencing systems. Fouss and Chang describe the main purpose of conferencing systems is to provide or enhance synchronous communication. Most co-present synchronous meeting groupware are based on Single Display Groupware (SDG) and What-You-See-Is-What-I-See (WYSIWS) applications.

\subsubsection{SDG}

Single Display Groupware typically includes a large display that multiple participants can interact with. These include the tabletop interfaces discussed earlier as well as other systems that are used to communicate and share ideas using a central shared display.

The Xerox Liveboard is a directly interactive, stylus based large area display [19]. Each member interacts with the board using a Liveboard Pen. This pen can be used to point from a distance and interact with the Liveboard by directly interacting with the display. A participant may possess his/her own Liveboard Pen or the pen can be passed around to hand over the floor.

The Pebbles Project focused on creating applications to connect multiple PDA's to a main computer [44]. The project investigated the use of such applications in the 
context of co-located meetings. PebblesDraw [45] was a shared whiteboard application that allowed all of the users to send input simultaneously from the PDA's while sharing the same PC display. RemoteCommander allowed users to control the PC's mouse cursor and keyboard input using a PDA.

Rekimoto also investigated the use of multiple mobile devices with a digital whiteboard [53]. With his system each participant possessed his/her own mobile device (PDA). This device was then used as a personal toolbox where various tools can be selected. The participant can then interact directly with the digital whiteboard using the tools they have selected.

\subsubsection{WYSIWIS}

Conferencing aids are applications used to enhance an existing conferencing system and are often part of desktop conferencing applications. The two most common examples of such applications are whiteboards and application sharing programs, which essentially use the WYSIWS method for collaboration.

Whiteboard applications present all participating users with a window in which they can place text and graphics information. As changes are made all user views of this window are updated in real-time. The window becomes shared scratch pad that allows users express, organise and share their ideas. Microsoft Netmeeting [42], Microsoft Live Messenger [41] and CU-SeeMe [16] have whiteboard utilities as components.

Application sharing programs are utilities that allow a user to activate an application on their desktop and share it with the people in the conference. Depending on the application sharing software other users may only be allowed to view the work or can 
actually be given control of the application. Microsoft Netmeeting [42] and Live Messenger [41] allow applications to be shared.

For application sharing, collaboration transparency systems provide the shared use of existing single-user applications. They are used to collaborate legacy applications that were developed with no support for collaboration in mind. These systems support synchronous collaboration. With these systems, each participant would have an identical view of the running application and an opportunity to interact with it. [7] [8] [26]

\subsubsection{Handheld CSCW}

Schmidt and his colleagues explored possible contribution of handheld computing in the field of CSCW and suggest new application domains in CSCW [60]. They focused on the extent to which co-located CSCW has been addressed and found that most CSCW research were focused on Remote CSCW. They proposed that handheld devices can provide new solutions for co-located collaboration in synchronous as well as asynchronous scenarios.

Schmidt et al. noted that the distinguishing feature of co-located co-operative work is that the participants are close enough together to communicate directly. Mobile technologies hold the potential to setup ad hoc and low cost computer supported meetings, not relying on dedicated rooms and environments. The requirement for meeting support technology can be lowered if personal devices rather than shared equipment constitute the user interface.

\subsubsection{Requirements for Photo Groupware}

Frohlich et al. have studied the requirements for groupware for sharing photographs [24] They take a look at the strengths and weaknesses of past and present technology 
to give recommendations on future photo applications they call 'photoware'. Four categories of photoware were identified based on time and place.

\begin{tabular}{|c|l|l|}
\hline & \multicolumn{1}{|c|}{ SAME TIME } & \multicolumn{1}{|c|}{ DIFFERENT TIME } \\
\hline SAME PLACE & $\begin{array}{l}\text { Prints } \\
\text { Slides \& projector } \\
\text { CO-PRESENT SHARING } \\
\text { Photo viewing software \& } \\
\text { devices }\end{array}$ & $\begin{array}{l}\text { Shoeboxes } \\
\text { Album \& frames } \\
\text { ARCHIVING } \\
\text { CD-ROM } \\
\text { PC filestore } \\
\text { Photo website \& Blogs }\end{array}$ \\
\hline DIFFERENT PLACE & $\begin{array}{l}\text { Telephone } \\
\text { REMOTE SHARING } \\
\text { Application sharing } \\
\text { Instant messaging } \\
\text { Video conferencing } \\
\text { MMS }\end{array}$ & $\begin{array}{l}\text { Mail } \\
\text { SENDING } \\
\text { Email attachment or website } \\
\text { reference } \\
\text { Internet photo frames }\end{array}$ \\
\hline
\end{tabular}

Table 2.3. Categories of photoware with example products and services - adapted from [24]

Frohlich et al. interviewed eleven PC-owning families about their use of conventional and digital photos. These families partook in completing photo diaries and recorded photo-sharing conversations over a three-month period. From this study they have identified that out of all the methods of interacting around photos, sharing photos in person (co-present sharing) was described as the most common and enjoyable. This was seen as a way of re-creating the past and reliving the experience with others who were there at the time. [24]

The families did organise their conventional photos in albums for social purposes or events. However, very few families reported systematically organizing their digital photo collection on the PC. The reason for this was that they never saw the PC screen as a convenient device for synchronously reviewing and sharing those photos with others. Many of their participants also reported being "turned off" by the notion of looking at digital photos on a computer screen when sharing with friends and family. [24] 
From their analysis of remote-sharing they have found that people are already successfully exchanging digital photos with each other asynchronously via e-mail, but reverting to the use of the telephone in order to discuss them live with remote partners. From this study Frohlich et al. recommend considering ways of building on the foundation of printed photo sharing rather than replacing it with forms of screen-based photo-sharing. [24]

\subsection{Summary and Design Implications}

Chalfen identified photographs to be social artefacts that are used to trigger memories and emotions. Sharing photos is a group activity which brings about the feeling of connectedness between those who the photos are being shared with. Conventional photo sharing where stories are shared with friends and family was defined as the Kodak Culture. This culture is important as the practices found here seem to be the most enjoyable social interactions when sharing photographs. The main interaction, photo-talk, includes storytelling and reminiscing. Crabtree and his colleagues identified three components for photo-talk, two of which can affect the design of our prototype. The first component is that photographs need to be organized in such a way as to be ready for retrieval. The second component is that the collection of photos should be easily manipulated.

With the development of digital cameras, the cost of taking photographs was no longer an issue. Portability of photos was drastically improved and various new methods and possibilities of sharing photos have been developed. The traditional way of sharing digital photographs was via the PC screen. Users typically did not organise their digital photographs as the PC screen was not seen as a convenient device for synchronously reviewing and sharing those photos with others. Online photo-sharing websites allow users to post photos and comments online to share with remote 
friends and family. It was found that sharing photographs this way was not as enjoyable as conventional photo sharing. Vronay and his colleagues tried to determine why sharing photos this way was not as compelling as sharing photos face-to-face. They found that sharing photos online with text annotation does not convey the emotion and storytelling as sharing photos face-to-face.

With the increasing popularity of mobile phones and advancements in camera technology, industry began integrating digital cameras with mobile phones. Consumers can now take digital photos at any moment they desire and send these images to their friends almost instantaneously. Research was conducted to determine the behaviours of photo sharing with these new devices. It was found that photos were not sent very often. This was due to the expense, complexity and poor image quality. When photos were sent to remote others, it was found that the stories told were mostly photo-driven rather than story-driven [31]. It was also established that mobile phones were being used as personal flip books and that most photos were shared with co-present people on the mobile phones screen.

Attempts at photoblogging from cameraphones were also pursued. Photoblogging systems provide a way to publish mobile pictures on the web and allow visitors to comment on published pictures. These services supported ambient virtual co-presence which gives users a sense of closeness by knowing what their social network of friends or family are doing, however this method of photo-sharing does not support photo-talk.

Existing photoware constrains the "communication of experience" which is the main reason of photo sharing. The printing of photos and the creation and organising of photo albums were identified to be for social purposes by inducing photo-talk. Photo-talk is best achieved and most enjoyable when sharing photographs 
co-presently with others. The design for our photo-sharing prototype will therefore build on the foundation co-present sharing.

Current digital co-present solutions included the tabletop interfaces and Balabanovic's Storytrack [4]. Tabletop interfaces are great for sharing digital photos, but lack the portability and no commercial tabletop interfaces are currently available. Balabanovic stated that to share photos in a natural setting, a portable device is required and should be large enough to show photos at a size similar to regular prints. The application that will be developed will be for a PDA device. The screens found on PDAs are generally larger than typical mobile phones and only slightly smaller than regular jumbo prints.

It was identified that gestures are an important component in storytelling. Gestures highlight objects for perception and draws attention to what is being talked about. Highlighting and drawing attention to objects will be an important aspect in the design of our application. PDA devices include a touch screen interface for photo manipulation, which we believe to be promising for supporting digital gestures.

Crabtree and his colleagues suggest that new interfaces need to augment and support the production of accounts, the telling of stories and thus support the conveyance of experience. Using this background research as our inspiration, it seems clear that the next generation of photo sharing application should allow users to share photos in an ad-hoc way without involving external pieces of technology such as computers or monitors or group displays. As most people carry a device capable of displaying digital images (in the form of a cameraphone) it seems sensible to develop an application that could broadcast an image amongst co-present handsets. Using a variation of WYSIWIS, this will provide flexible group view for all participating users. WYSIWIS applications are naturally synchronous and sequential which we believe to 
support the very nature of photo-sharing and photo-talk. This application will fit in the handheld groupware classification and will be co-present and synchronous in the CSCW time/space matrix. Assuming that this can be achieved technically, the problem remains of broadcasting images in such a way as to support the type of social interaction that users experience with print photographs. 


\section{Design and Implementation}

\subsection{Background}

Recently, more mobile devices are defaulting to Wi-Fi and touch-screen technology as standard. Many of the new Nokia N and E series include Wi-Fi. Using Wi-Fi, data can be transferred quickly between multiple co-present devices and also be used to connect to the internet. Some fully touch-screen mobile phones include the LG Prada phone and the Apple iPhone. Touch-screens allow for changeable interfaces and additional types of input such as pointing at icons on the screen and drawing. The iPhone as well as many Windows Mobile phones include both touch-screen and Wi-Fi capability. We have thus chosen to use PDA devices with fully touch-screen displays and Wi-Fi capability as handset surrogates.

\subsubsection{WYSIWIS}

Berry et al. point out that collaboration often relies on all group members having a shared view of a single user application [8]. WYSIWS (What-You-See-Is-What-I-See) applications allow a group of users to share an identical view of a running application. This allows for collaboration where each participant has an opportunity to interact with the application. Greenberg [26] discusses the responsibilities for view-sharing software that must be considered during its design and evaluation. These responsibilities include maintaining consistent shared views, managing floor controls between participants wishing to interact with the system and allowing participants to gesture and annotate around shared views.

In order to bring collaboration to these applications, each participant should be able to interact with the application. Greenberg mentions that social protocols is a reasonable way of controlling casual sharing of applications between small groups of about 2 to 3 
participants, provided that the consequences of accidental simultaneous interactions do not lead to major complications. More advanced floor controls are suggested as the groups become larger.

In the literature, there are two main types of WYSIMS systems described [7][8]. Strict WYSIWS forces all viewers to share the exact same view that the presenter sees despite their role in the group. Berry et al. mention that this conflicts with the different needs of the presenter and audience. Relaxed WYSIWS includes a public and private space as you do not always want people to see everything displaying on your screen, thus depending on your role, all participants will have similar but non identical views.

Brinck and Gomez [10] studied the contents of office whiteboards after they had been used in conversation to better understand the use of artefacts in communication. They discovered that whiteboards are used to present and discuss various classes or artefacts that they call "conversational props". The whiteboard was seen as a medium in which these props are introduced and manipulated. This study lead to the development of a prototype remote WYSIWS multi-user drawing tool called the Conversation Board. This was essentially a shared whiteboard application designed for geographically separated people. The Conversation Board allows each participant to have his/her own custom view (relaxed WYSIMS). Only the canvas is shared identically between all users.

From their studies, Brinck and Gomez also found that typical office conversations involving a whiteboard included only 2 or 3 participants. They point out that it is unclear whether this will continue with digital whiteboards as physical interference is no longer an issue. The limitation to the number of users partaking in whiteboard conversation is limited by the computational power and a need for good response 
times. For WYSIMS collaboration, policies to determine when and what participant can interact with are needed.

\subsubsection{Coordination Policies}

The coordination among participants and the control of collaborative tools largely rely on conscious following of social conventions/protocols [37]. These conventions seem to be largely implicit but usually well understood by participants [9]. Social groups are coordinated by these conventions/polices. These polices are implemented in groupware as rules called "coordination policies". Coordination polices include rules to determine access control, concurrency control and floor control. Li et al. point out that many of these social rules are better built into the software as mechanisms to ensure that they are followed by all collaborating participants while some others are better left to the participants as social protocols to obtain more flexibility [37]. Social protocols are used in face-to-face encounters. They are flexible and encourage collaboration but it is difficult to guarantee that they are followed. Li and his colleagues found that this is not a major issue with small face-to-face group meetings especially when people are familiar with each other [17]. Boyd described floor control as the problem of managing interaction among users of an application [9]. We are interested in the interaction that occurs when using our prototype, and thus will focus on floor control policies.

Dommel and Garcia-Luna-Aceves defined floors as "temporary permissions granted dynamically to collaborating users in order to mitigate race conditions and guarantee mutually exclusive resource usage" [18]. Floor control polices deal with conflicts within shared workspaces by regulating turn-taking. It is a user-centred concept and implementations should follow user expectations and the constraints imposed by the 
system and the network. The ultimate goal of floor control policies is to allow for maximum synergy in the cooperation among subjects [18].

Floor control polices are divided into 2 categories. Explicit policies require participants to deliberately request and release control. Implicit policies automatically requests control for participants, triggered when a participant generates input events. Crowley et al. [15] described 4 variants.

- Explicit request with explicit grant

- Explicit request with implicit grant

- Implicit request with explicit grant

- Implicit request with implicit grant

Out of these 4 variants all are explicit policies, except for the last. Dommel and Garcia-Luna-Aceves suggest using explicit policies for real-time interactions and implicit policies for asynchronous task completion. They also mention that the absence of any limitation on the floor-holding time could result in competition for floors resolved by social protocols [18].

Greenberg presents some examples of floor control policies described below [26]:

- Ring passing: The current host must explicitly release control before anyone else can assume it. Tension can arise between a participant who will not release control and others who wish to acquire it.

- Pre-emptive: Any participant may grab control of the floor at anytime. This can lead to excessive interruptions in an aggressive conference. 
- Time slices and time outs: A person may have a set time-slice for control after which the floor is taken away from him. The floor can be released for anyone's acquisition if the current controller has been idle for a time period.

- Moderated: A designated participant may act as a chairperson who is responsible for passing control to the other participants.

\subsubsection{Notworking}

Wireless interconnectivity is becoming something of a de facto standard on mobile devices. Most current handheld devices have the ability to wirelessly connect to other devices within close proximity, a feature explored in a number of research projects such as Push!Photo [54], Push!Music [29], TunA [6] and Collaborative Games [57].

Through ad-hoc networking, Push!Music and Push!Photo share music and photo files with other users in proximity. TunA is a mobile peer-to-peer application that allows users to share their music locally in a synchronised way. It allows users within the Wi-Fi range to connect and listen to the same song at the same time. The method of connectivity is via an ad-hoc $802.11 \mathrm{~b}$ wireless network connection. The implementation of collaborative games in [57] also connect via a peer-to-peer wireless ad-hoc networking using the OpenTrek platform [58]. From this research, it seemed that using Wi-Fi ad-hoc networking was quite flexible and efficient and thus we pursued this technology to implement our prototype.

\subsubsection{Multicasting}

If the same data needs to be sent to multiple recipients, a typical packet would need to be sent individually to each user. This approach produces a lot of overhead and the latency increases with the number of recipients. For real-time data, this performance 
is not acceptable. Multicasting is the transmission of a packet to a subset of the hosts in the network. Multicasting offers two important benefits to network applications [37].

- Efficient multi-destination delivery

- Robust unknown destination delivery

Each multicast address identifies a host group. A group of hosts listening on this address will receive a packet sent to that address. These packets are delivered with "best efforts" datagram reliability. The service interface imposes no restriction on the number or location of hosts in a group. Hosts can join and leave groups at will, with no need to synchronize or negotiate with other members of the group or with potential senders to the group. [17]

Li et al. proposed COCA (Collaborative Objects Coordination Architecture), a generic framework for developing evolvable collaborative systems based on IP Multicasting [37]. This architecture was developed for desktop PCs on a wired network. COCA allows for various roles and rules to be defined for each participant. The rules define the coordination policies which can easily be changed thus allowing the policy to easily evolve. A moderator role is required to manage floor control. These defined rules are interpreted by the COCA Virtual Machine (VM) which $\mathrm{Li}$ and his colleagues point out to be a potential problem with this architecture. All messages and media streams to and from each participant will run through the COCA virtual machine which could cause a decrease in performance. This architecture seemed to work well, but one problem mentioned with this architecture, is that packets are delivered with "best effort" reliability and thus packets are not always delivered. 


\subsubsection{Reliable Multicasting (RM)}

The main problem identified with multicasting is that packets sent are not guaranteed to reach their destination. In literature, there are a number of techniques that can be used to overcome this inconsistency in acceptable bounds.

TCP (Transmission Control Protocol) provided reliability of typical unicasting. It has a point-to-point model of communication and is impractical to modify for multicasting. TCP works by sending acknowledgement packets (ACKs) containing an identifying sequence number for every packet correctly received. Implementing this technique in multicasting could result in an "ACK implosion" [20] that would overwhelm the sender with ACKs from the many receivers in the group. There is currently no "one size fits alln reliable transport protocol for multicast as different multicast applications have widely different requirements for reliability. Pullen suggests mechanisms that can be combined to achieve RM under specific circumstances. [52]

- Continuous update of state data at frequent intervals: reliability comes from the built-in redundancy in human interpretation of the media.

- Negative Acknowledgement (NACK)-based reliability: Any practical network will deliver successfully many more packets than it drops, so the first step toward RM is to use a negative acknowledgement when data is not received, rather than a positive acknowledgement when data is received.

- Heartbeats: A problem with NACKs is that, unless messages are sent at regular intervals, there is no way of knowing it has missed a message until a subsequent message is received. A solution to this problem is to send a periodic "heartbeat" message at a low rate. The heart bears the sequence number of the last message sent, and is used to trigger a NACK where appropriate. 
- Retransmission: The requirements for RM implies that at some point the lost data must be transmitted again. In multicast file transfer it is possible for the NACKed messages to be retransmitted at the end of an initial, complete transmission of the file.

Ngadi and Doherty [46] and Floyed and his colleagues [22] implemented and prototyped a distributed whiteboard application using reliable multicast techniques. These systems proved to be scalable, portable and had reasonable performance. From this research, multicasting seems promising as a viable solution to use in the application we intend to develop.

\subsubsection{Design Implications}

From the literature surveyed, it seemed that our conceptual design for an application that can broadcast an image amongst co-present handsets is possible. This application will use a form of relaxed WYSIWS to share a display of photos. The photos will be broadcast to other handsets using IP multicasting. From our background research, it seems that social protocols have been observed to be effective in small groups of about 2 to 3 people or groups where people are familiar with each other [10][27][17]. Li et al. also mentions that social protocols can add more flexibility. For this reason, we will implement a two floor control policies (implicit and explicit) as well as include a "free-for-all" mode to determine if social protocols are effective.

\subsection{Interface}

Using a PDA to act as a cellular handset surrogate, the prototype was developed for HP iPAQs with build-in $802.11 \mathrm{~b}$ wireless cards using the .NET compact framework 2.0. The fundamental aspect of this application would be an interface to view and 
browse through photographs that are wished to be shown to others. The HP iPAQs include a photo viewing application called "IFAQ Image Zone". This application has a thumbnail browser at the bottom of the screen that displays all the images stored in a specific folder. When an image from the browser is selected the full version of the image is displayed on the main display area of the application.

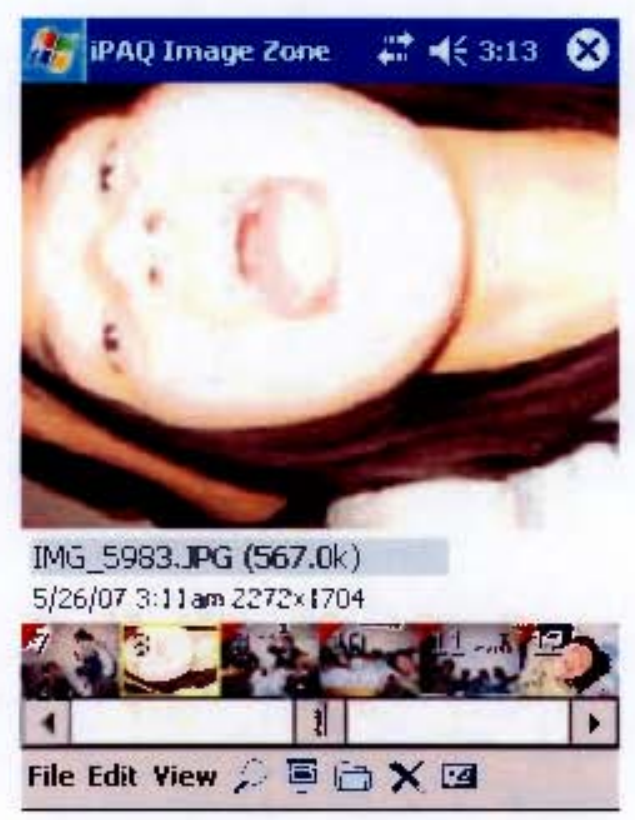

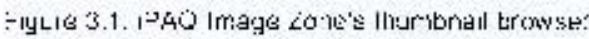

When the main display area is tapped. the application switches to another mode that allows you to explore the image in more detail. Various tools are provided that allow the user to zoom and pan through the image. In this mode there is also a navigational thumbnail that appears for a few seconds after an input event. This navigational thumbiail notifies the user of which section of the photo is being viewed via a square drawn around the area being displayed. The user can also point and drag this square around the thumbnail to view different sections of the image. 

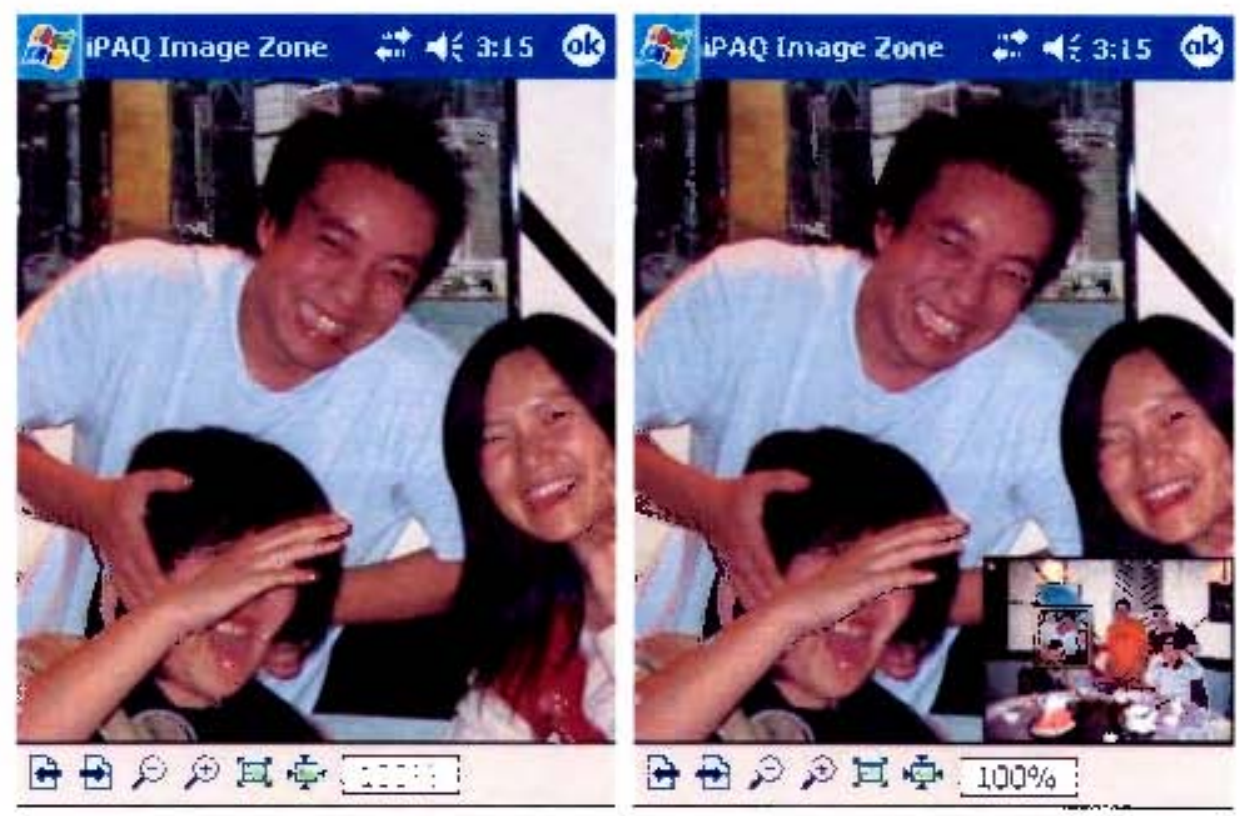

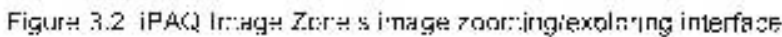

Our interface will use the essential components of $\mathrm{PAQ}$ I mage Zone to navigate through photographs In our initial interface prototype our toolbar included buttons for the user to exit. change options (device name and multicast addressi, change folder, connectidisconnect from a broadcast, request host hides show thumb nail browser: hideishow image tools and change to full screen mode

The thumbnail browser is based on that of iPAQ Image Zone. When a thumbnail is selected, it is displayed on the main display area. Users can zoom in and out of images and pan them by dragging the stylus on the maın area. A navigational thumbnail was also implemented to assist with panning the image and notifyıng the user of the section of the image that is currently being displayed in the main display area.

Brinck and Gomez identify that gesturing is somethıng that is often done in context of face-to-face conversation [10]. They note that people often used sketching in order to gesture. This is supported by Crabtree et al. who suggested supporting gestures by implementing 'telepointing' and 'telepainting' [14]. A pointer that allows users to point 
at various objects on the screen as well as the ability to draw on the photographs was implemented to support gesturing.

To connect to a multicast group, the connect button must be pressed $(\vec{\sigma})$. When connected, the degree of WYSIWIS only extends to the photo displayed on the main display area. Participants have unique views of toolbars, image manipulation tools and thumbnail browsers.

For each user, only the main display area will act as a WYSIWIS canvas. The thumbnail browser will only show photographs stored on the users device. Each participant can choose to hide/show his/her own thumbnail browser and toolbars.

\subsubsection{Heuristic evaluation}

A heuristic evaluation was conducted on this prototype interface with usability experts. The result was minor changes made to the interface.

- The thumbnail browser initially displayed six thumbnails across the width of the screen. These were identified as relatively small for a user to identify the details of a specific photo. The application was modified to display four photos across the width of the screen, and thus present larger thumbnails.

- The loading of thumbnails while scrolling/browsing through images was also identified to be a bit slow and would thus seem unresponsive to the user. Pre-rendering the thumbnails on load time and storing them in a cache was implemented to solve this problem.

- The bottom toolbar included two icons that represented drawing tools and zooming tools $(\mathcal{P})$. These icons were identified as possibly confusing for a user. This was changed to a single icon ( $\mathcal{D})$ to represent all image tools. 
- The rotation icon was seen as possibly confusing as it was not the standard rotation icon used in most applications. This icon was changed from this (C) to this $\{=1$ ).

- The "ciear drawings" icon was initially represented by a squeegee and a spray bottle (7). As the size of an icon was 16 by 16 pixels, this could not be illustrated effectively and was confusing. This was changed to represent a simpler "car window wiper" ( 7 ) as the icon.

Figure 3.3 shows our interface after the heuristic evaluation. Refer to Appendix 1 for a more detailed interface walkthrough.

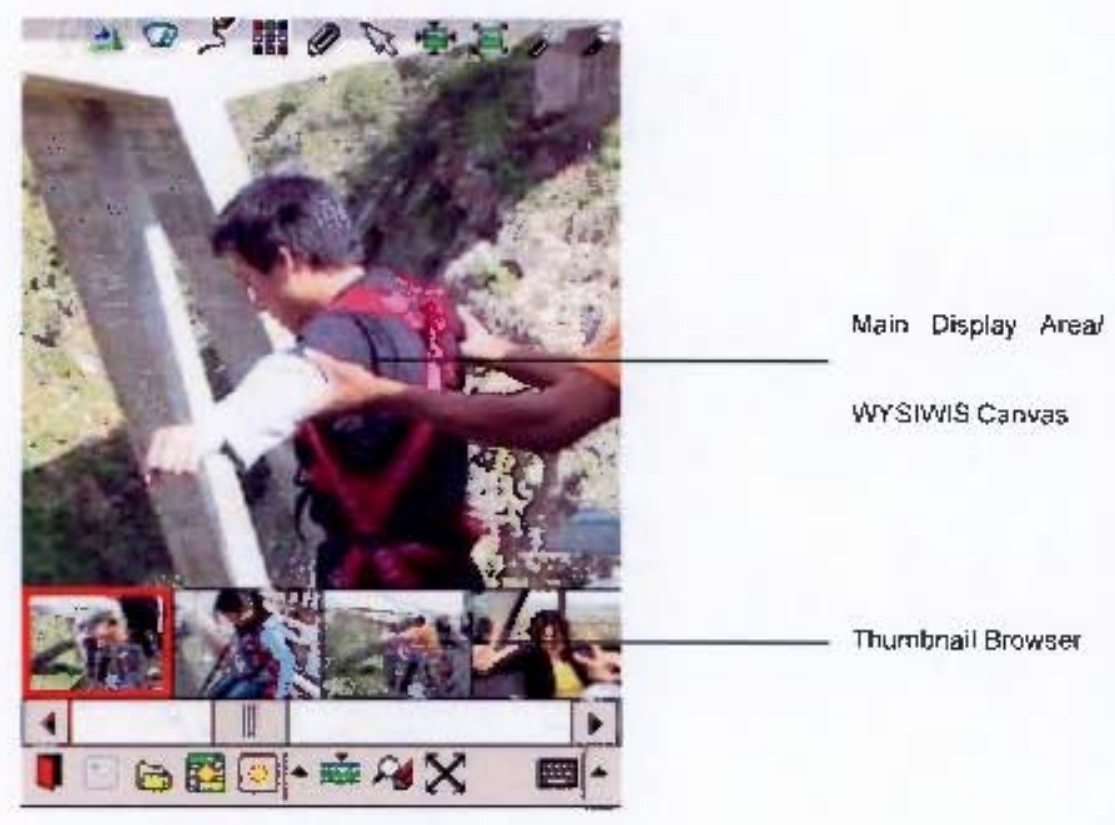

Figure 3.4. Protoly pe interface after hesristic evaluation

\subsection{Floor Control Policies}

The user that is in control of the display broadcast is determined by a floor control policy. Begole et al. indicate that explicit policies diminish participants feelings of involvement in the collaboration as it makes turn-taking apparent and interrupts the flow of activity [7]. This is echoed by Greenberg who describes explicit floor control 
mechanism schemes as unduly contrived and restrictive [26]. We chose to implement three floor control policies to determine which type of policy best fits the requirements for photo sharing. We chose to implement one explicit policy, one implicit policy and a free-for-all mode to determine whether social protocols can be effective in this highly social interaction. Dommel and Garcia-Luna-Aceves suggest that when using floor control policies, current control status needs to be shown [18]. For all of the implemented floor control policies, a method to identify floor control status has been implemented. Dommel and Garcia-Luna Aceves also mention that certain properties or functions could be exempt from being floor controlled depending upon users or the session purpose. Photo sharing naturally leads to people pointing at things. We considered the ability to point at objects on photos without having to hold the floor important: thus we have allowed the pointer to be able to be controlled by all users whether they hold the floor or not.

\subsubsection{Host-Token}

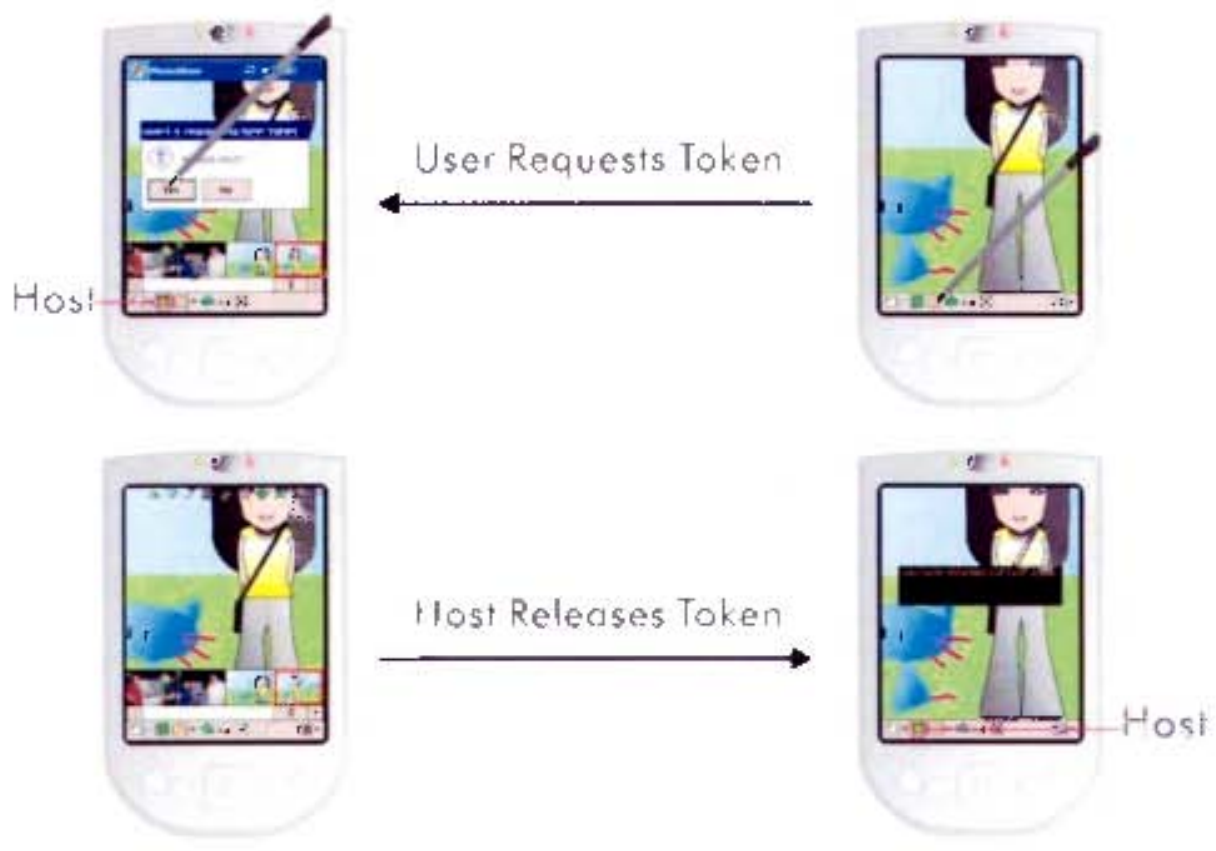

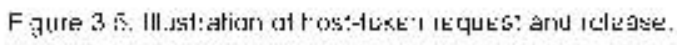


With this policy. the user that possesses the host token has full control over the WYSIWIS canvas. If a user is listening to the broadcast. this is identified by a green connect button (局). If a user possesses the host token, the connect button changes to this icon (履). The user that initially possesses the host token is the first user to join the show. This token can be requested by other users by pressing the "request host" (ㅇ) button. The hast will then receive a message to notify himiher that a user is requesting the host token. The host can then either releaseideny the host token. This is an explicit floor control policy and reflects Crowley's explicit request, explicit grant variant.

\subsubsection{Three-Second Policy}

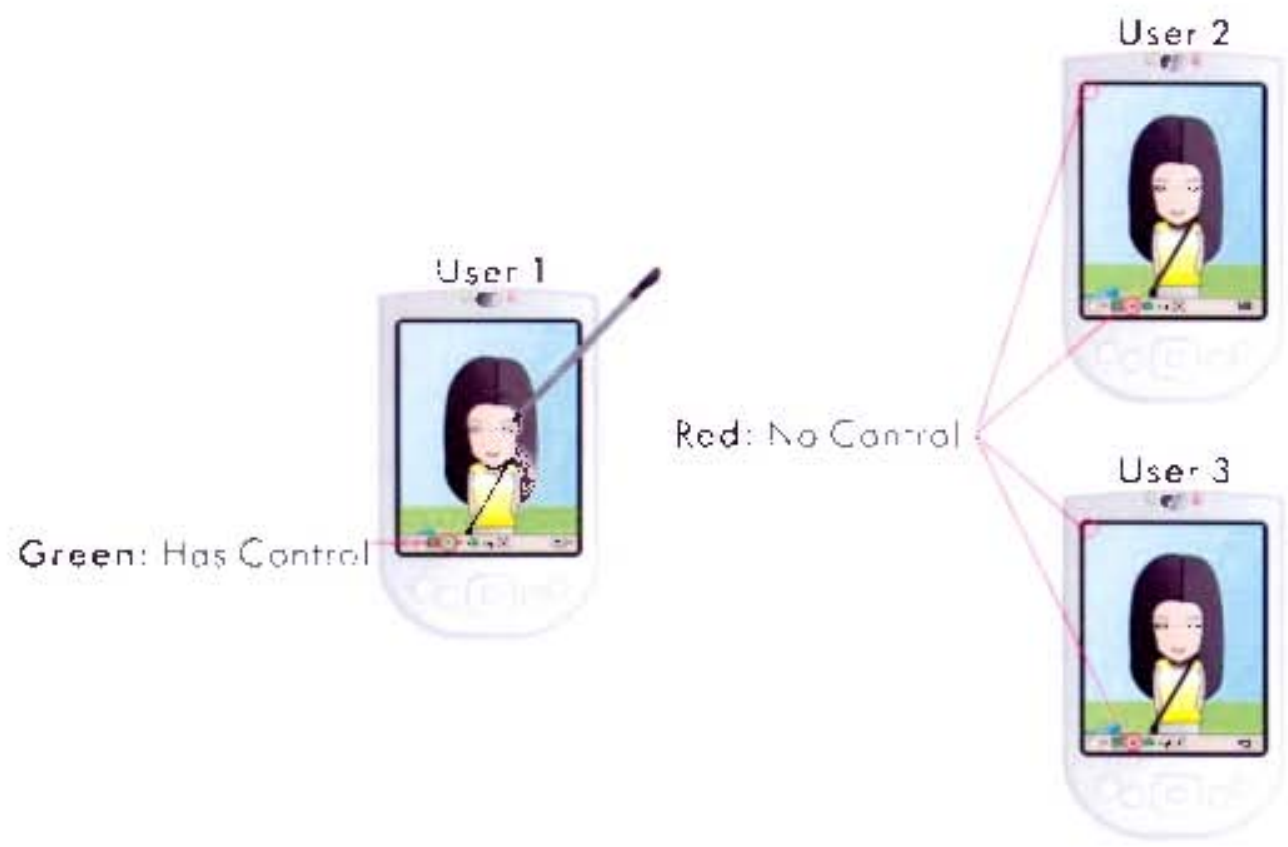

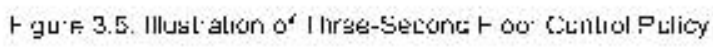

With this policy, inilially a user is given control of the display once heishe performs a control action. A control action is any action that would manipulate the display (i.e. pan, zoom. rotate, draw, etc). This user will retain control for three seconds after his her last control action. If no control action is performed for three-seconds. another 
user can take control of the display by performing a control action. Users can identify when they are biocked from taking control by a notification icon that is displayed in the corner of the screen. When this red circle is displayed, it means that the user is blocked from performing any control action. When no circle is displayed, it means that heishe is free to manipulate the display. The button used to "request host" in the host token protocol also changes to reflect control information. A red icon ( 9 ) means that control is blocked for the current user. A green icon (t) means the user may now attempt to perform a control action. Three seconds was chosen as in an initial pilot, this was the maximum length of time users could look at an image before becoming bored. This is an implicit floor control policy and reflects Crowley's implicit request, implicit grant variant.

\subsubsection{Ad-hoc Policy}
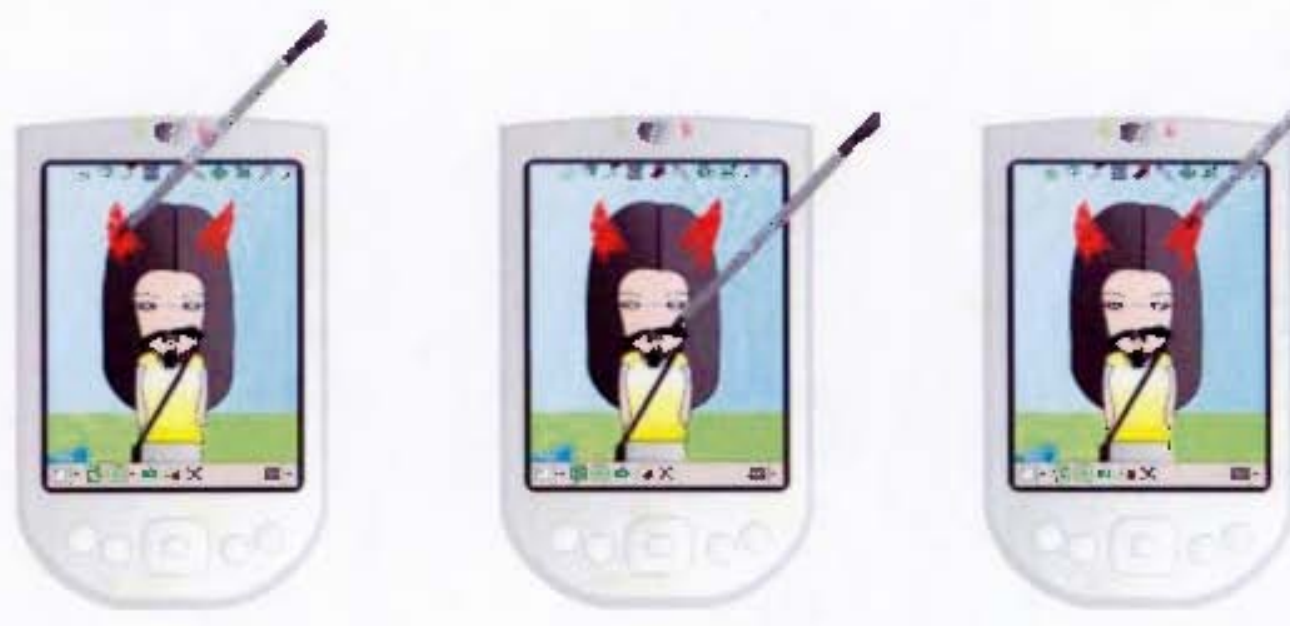

Figure 3.7. Ad-hoc Policy, all users can simultaneously send control data

With this policy any user can control the display at anytime. The motivation in implementing this protocol was to determine if users can coordinate control socialiy without any software locks. It is anticipated that a social protocol would emerge to organise any chaotic behaviour that is expected. 


\subsection{Implementation}

Synchronising the display of all listening devices was achieved using IP multicasting. Devices can connect/disconnect from the show at any time. Other devices are not affected as multicasting does not require devices to know about other listening devices. A messaging system was developed to co-ordinate floor control using this method of communication. Floor controls are all managed within independently running instances of our application.

When a device connects, a message is broadcast to determine if there is any current host on the multicast IP chosen. If no host responds, the device declares itself as the host. If a host is found, the device is sent the current show data to synchronise with the show. The host of the show can select which floor control policy to use.

When the host-token policy is active the device that possesses the host token is allowed to broadcast photo and control action data. When the three-second policy is active, this causes devices to be blocked from broadcasting data for three seconds after the last control action packet has been received. Using the ad-hoc policy, photo and control data can be broadcast from any device at any time. Messages from all users are merged into a single stream on a first-come, first-served basis. Greenberg suggests that this is the simplest approach. He notes that this seems chaotic, but can work well in practice [26].

It is anticipated that photo manipulation data will be sent frequently. Reliability will be achieved using this frequent stream of data and including complete photo orientation data in each packet. Host request/release packets are sent multiple times to increase reliability. This seemed to work well in preliminary trials. File transfers without reliable mechanisms were implemented. Files seemed to be sent in a reasonable (1 second) amount of time. Since multicast has "best effort" reliability, occasionally photos would 
be corrupt due to lost packets. From the literature surveyed, it is recognised that reliable multicast file transfer is possible. We have not implemented this as we can simulate reliable file transfer by copying the photographs on to all the devices before running the experiments.

\subsection{Summary}

A simple prototype has been developed to broadcast photos and control actions. Using multicasting, the WYSWS canvas is synchronised with acceptable performance. Three floor policies have been implemented, explicit, implicit and "free-for-all". In our next chapter, we will evaluate these policies and how they affect interaction amongst a group of friends. 


\section{Evaluation}

\subsection{Background}

Evaluation is used to understand how a product is/will be used in its intended setting. Observation (watching and listening) techniques can be used to evaluate prototypes. Preece et al.[51] mention that the key aspect of evaluating is involving appropriate users. For laboratory studies, these users must be found and screened to ensure that they fit the intended target population. The tasks that are assigned to these users should also represent those for which the prototype was designed. Prior to running an experiment, a trial run/pilot study should be conducted to identify any potential problems in advanced. [51]

It is important to have goals and questions to guide an evaluation and give focus to an observation. The data that is collected is qualitative and requires understanding users by behaviour patterns observed [51]. Evaluating collaborative systems designed for entertainment is challenging as success is not defined in terms of productivity and performance, but in terms of enjoyment and interaction [40]. Mandryk and Inkpen [40] studied collaborative computer games and found that these systems support interaction with the system rather than with each other. They note that even when players sit side-by-side, they still tend to interact with each other via the interface.

Mandryk and Inkpen state that emotion is important to design when the primary goals are to entertain the user and that traditional objective measures used for productivity environments such as time and accuracy, are not relevant to collaborative play. They note that recording gestures, body language, and verbalizations is a rich source of data which can be used for evaluation. [40] 


\subsection{Experiment Design 1}

\subsubsection{Experiment Design}

The objectives of our experiment are to determine

- whether our prototype can support interaction and communication between participants to create engaging experiences

- what social behaviours this application will produce/encourage

- how floor control policies affect the social experience

To achieve these objectives, it was decided that a naturalistic observation would be conducted. Naturalistic observations are typically used to observe users in their natural environment/setting [51]. For our experiment, we will use a controlled setting and observe how users would naturally use our system without requiring them to complete any specific tasks.

Our prototype was designed to support photo-talk. Frohlich [24] notes that storytelling photo-talk occurred in conversations where photos were shown to others who were not there at the time the photos were captured and hence did not share the memory represented by the photos. Crabtree et al. [14] and Frohlich et al. also found that sharing photos exclusively with members of the original 'capture group' resulted in reminiscing talk where it is rare to find members of the original capture group to re-tell stories that they have experienced together. From these studies it was decided to use groups that consisted of four friends, three of which shared an experience together and captured a reasonable amount of digital photographs. The fourth friend would not have shared the same experience and had not seen the photos before. We chose only one friend to be excluded to maximize the number of photos that were available for sharing. The use of friends within a group will allow us to investigate the effect of 
the system as realistically as possible. Using this combination of participants, we hoped to provoke both reminiscing as well as storytelling photo-talk.

The groups were asked to bring about 60 photos of the experience that the three friends have shared. For this experiment, the group was to talk about their photographs with each other. Users were observed using video cameras and human observers in the room. Prior to the experiment, a tutorial was conducted to show the users how to interact with the application. They were also been given some time to explore the application on their own.

For the experiment, each user was presented with their own Pocket PC. The photos were preloaded on their devices. The users were told that they would be given about 15 minutes to talk about their photos using each floor control policy. The experimenter would notify them when the floor control policy was to change.

For each group, the floor control policies were tested in different orders as to eliminate any learning effects or fatigue effects for a specific policy. After the experiment, a group discussion was held to ask the users various questions on how they felt about the application and the various floor control policies.

Prior to all our experiments, the participants were told that they were being recorded. Concession forms were given that stated what the recorded data would be used for. Participants were notified that it was the system that was being tested, not them, and that they are free to leave at anytime during the experiment if they are feeling uncomfortable for any reason. 


\subsubsection{Pilot}

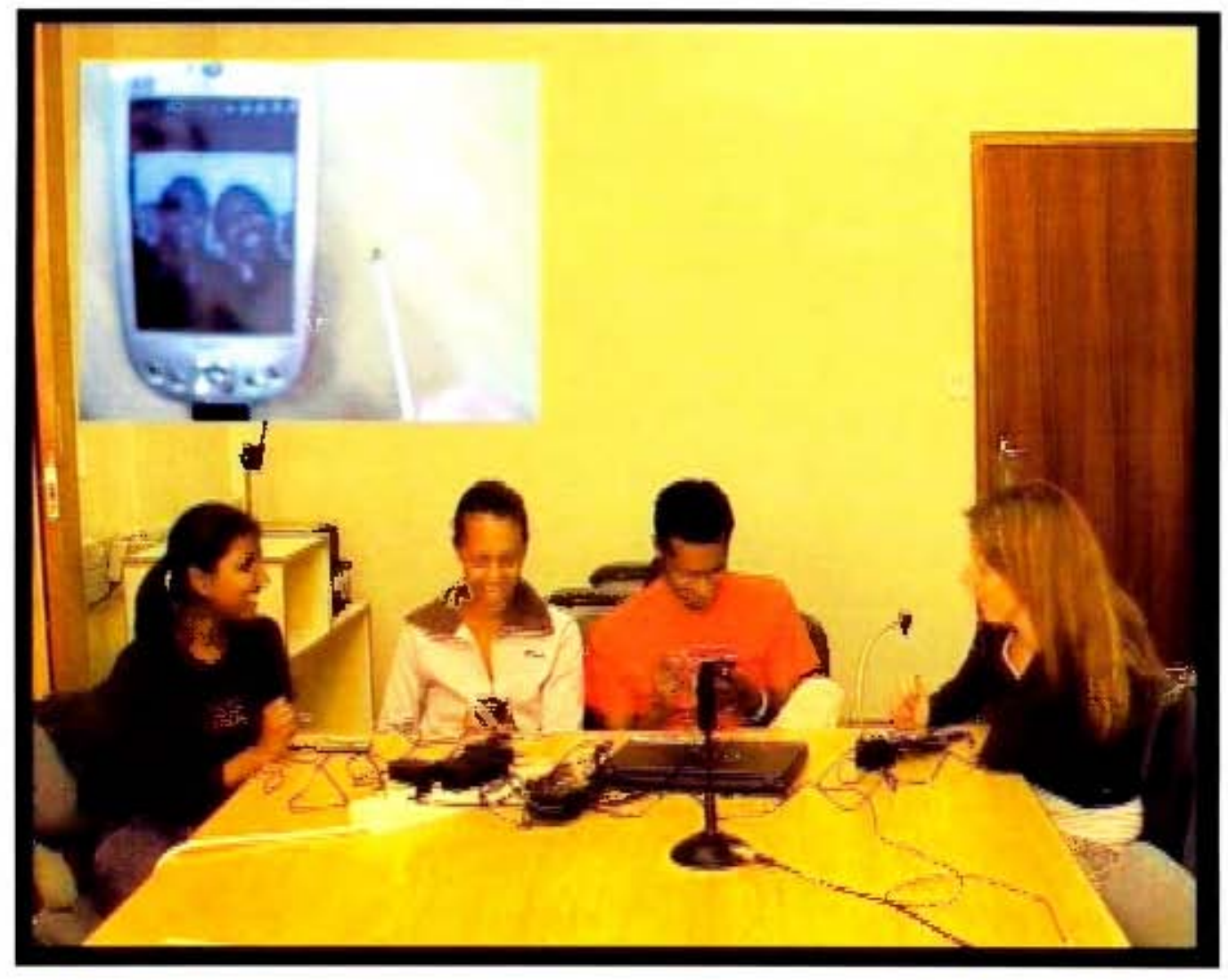

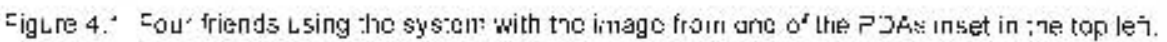

The pilot group consisted of friends that went on an overseas trip together. They were all university students and all owned a camera phona. During the tutorial, there were no difficuities from the users in understanding how to use the application or the floor control policy concepts.

From the pilot, the fourth friend. that was not part of the original capture group, assisted in generating storytelling photo-talk as intended. Collaborative storytelling and occasional reminiscing talk was also observed.

There were some minor issues identified relating to the interface with regards to the access of the exit button and connecting'disconnecting from the show. These buttons 
were placed on the main toolbar and were accidentally pressed from time to time. A slight modification to the interface was made, to make these actions less accessible.

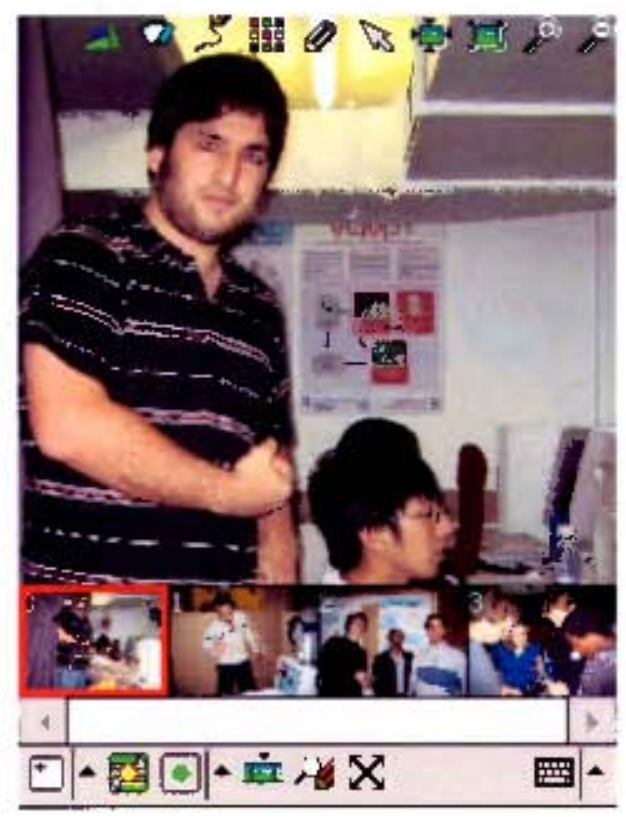

Figure 4.2. Refined prototype interface.

\subsection{Experiment 1}

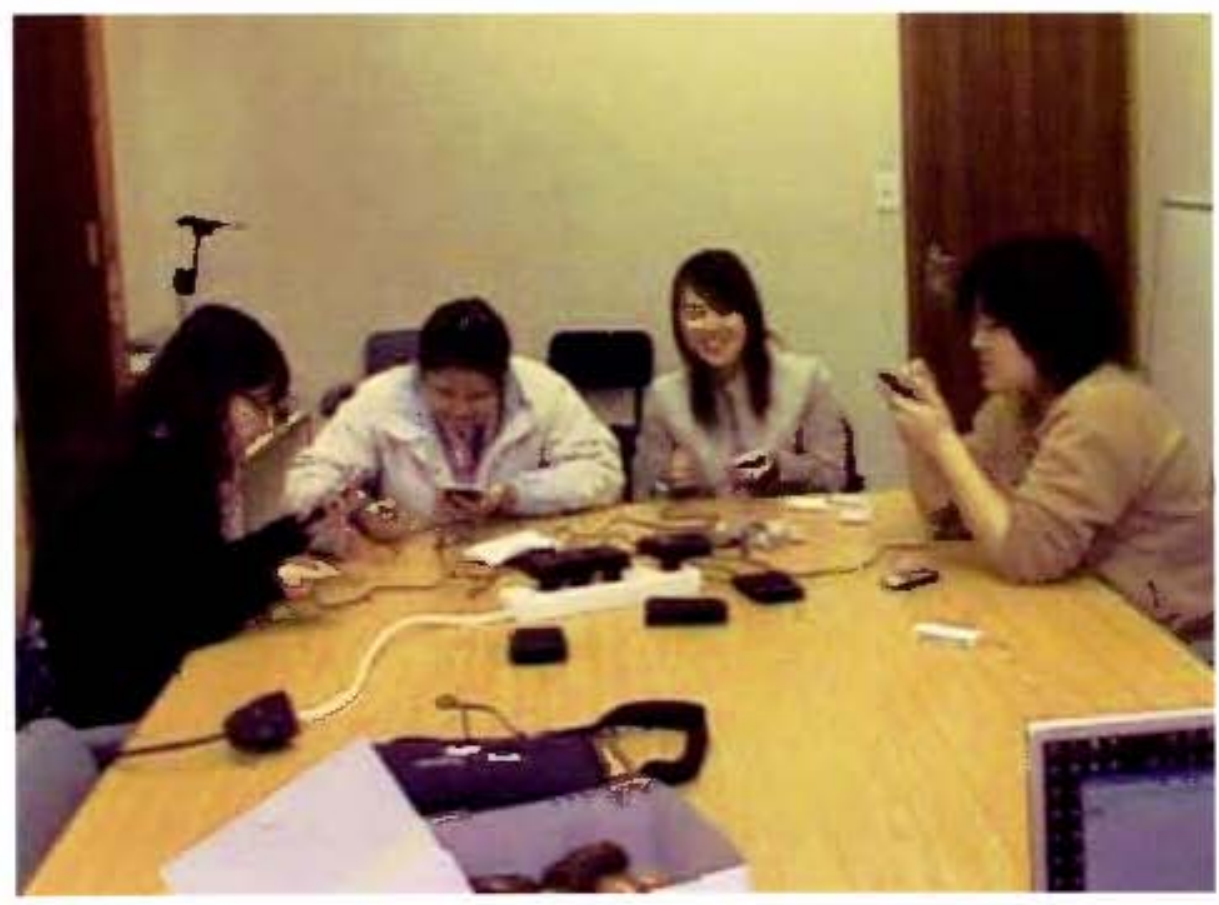

Figure 4.3. A group for first user experiment. 
The groups consisted of various combinations of males and females in their late teens or early twenties. The photos that the groups used were of a day outing, rock climbing and a party. With each of the groups, a user started by taking control of the display and storytelling their photos to the fourth friend that was not part of the experience. While the user conducted the storytelling, others in the group attempted to explore if/how they could contribute.

\subsubsection{Host-Token}

With this policy, the storytelling of photos and control of the display was very organised. Users seemed to enjoy having full control over the display, storytelling their photos without worrying about being interrupted. With this policy, collaborative storytelling observed by Frohlich et al. [24] did emerge. When a member of the original capture group wanted to add a comment to the story, he/she would ask the host to manipulate the image on the display to highlight what they wanted to comment on. When the fourth friend wanted to ask a question relating to an object in the photograph, he/she would also ask the host to manipulate the image to focus on what he/she wanted to ask about.

Some interesting behaviour was observed when passing the token. Users tended to pass the token verbally/socially. When the host was notified that a user was requesting the host token, he or she would ask who requested the host and usually deny the request. If the host was verbally asked for the host token, the host was usually more likely to release the host token. Participants found it entertaining to obtain the host token as it gave them full control over what their friends see.

Some users wanted to release the host token before another requested it, since he/she did not want to control the display any longer. However this was not possible as the method of passing the host token was not implemented this way. This led to the 
host asking other participants whether they wanted the host token. With one of the groups, another problem was observed with the host token policy. One user constantly tried to obtain the host token by constantly requesting it. The host did not want to release the host, which meant he had to constantly deny the request. This seemed to irritate the host, which eventually led to him releasing the host to the requester.

\subsubsection{Three-Second}

Storytelling persisted using this policy; however, the user telling the story and the user controlling the display were occasionally two different people. This was due to the automatic release of control three seconds after the last control action of a temporary host. Some participants gained control without wanting control, as they constantly had their stylus on the screen. Users that wanted to control the display seemed to start getting frustrated as they were always being denied the control. Users would wait for the notification when control was released and try to manipulate the show, only to realize someone else obtained control before them. This led to the users asking whoever was in control to manipulate the display. When a user wanted to take full control over the show, he/she would ask the current host for control. The host would then pronounce that he/she has stopped manipulating the display and that the requester can take control. When verbally asking for control, other participants in the group did not try to grab control. One group found it entertaining to keep control of the show, and would constantly manipulate the image so that he/she did not lose control. This group found keeping control highly entertaining and named this "game" that emerged "battling". 


\subsubsection{Ad-hoc}

Using this mode, the participants all tried to manipulate the image and found this mode very chaotic. Users tended to stop storytelling about their photos and instead everyone started to try and manipulate the display as much as possible. This was generally in the form of drawing on the screen, typically trying to tease a co-present friend that was in the photo. The friend being teased would then try to find a photo of the others, or draw on the other co-present users captured in the photo as a response. Since there was no software lock to determine who had control over the display, all users could simultaneously manipulate the display. This seemed very chaotic. However, within this chaos, all the users seemed to be enjoying this form of interaction the most.

\subsubsection{Group Discussion}

The majority of the subjects typically did all their photo activities (viewing and sharing) using a PC. This included viewing photos with their friends via the PC screen and sharing photos on the internet (online photo albums, blogs, e-mail). The interface of the application was found to be easy to use - they mentioned that the icons used to manipulate the display seemed familiar and thus intuitive. All the users enjoyed the synchronized shared display aspect of the application and agreed that they would use this type of application in real world situation. The only concern was the compatible devices for using this application. Drawing on the photographs was the most enjoyed feature of the application.

Almost all the users preferred the host-token policy as with this policy users have the most control of the display. They disliked the three-second policy as they did not know who was in control, and they could not always obtain control when they desired. They mentioned that with the ad-hoc mode, things were too chaotic. Even though the 
host-token policy was preferred, all users agreed that a choice of floor control policies would be best as the other policies could be useful for other situations.

The users were asked how this method of sharing photos compares with sharing printed photos. Most users did not print photos very often. If they did, they typically only print the more meaningful photos. Using this application, they could also share the photos that they would not normally print. The only criticism mentioned of using this method is that the resolution and quality of the photo displayed is not as clear as a printed photo. The size of the display is also not as large as a typical printed photo. However, these factors did not seem to detract overly from the users' enjoyment.

\subsubsection{Discussion}

\subsubsection{Host-token}

From the post-experiment discussions, users stated that they preferred the host-token policy. The reason being that control was explicit and removed any ambiguity (i.e. they have full control over the display). From our observations, storytelling and conversations were very controlled and happened in a civilized manner. There was little confusion on determining who was in control during the interactions. The only minor problem noticed with this policy is that users wanted to give up the host token before another user requested it first. This could not be achieved as the host token can only be released when it is requested. Our model requires that someone be in control and that the same someone must give up control. To allow voluntary token abandonment would mean that there is no one in control, allowing ambiguity to creep back in.

With this policy, a combination of story-driven and photo-driven behaviours were observed. The users' photo set was typically in a chronological order in the thumbnail 
browser. The user initially in control would start by displaying the first photo in the thumbnail browser. They would then start describing the photo being displayed on the screen (photo-driven). The next photo in the browser would then be selected and the photo described again. While describing a photo, a specific story would come to mind of the user. The user would then find the specific photos in the thumbnail browser to display to enhance the storytelling (story-driven). The photos following that photo were usually related and were displayed to continue with the story.

Using this policy collaborative storytelling did occur, however participants that wished to add narratives could not manipulate the display themselves. This resulted in these participants asking the host to manipulate the display.

From the observations and discussions, it is clear that this is the preferred policy for storytelling behaviour.

\subsubsection{Three-second}

The main problem found with the three-second policy is that users could not obtain control when they wanted to. The user currently in control was also unidentifiable unless users resorted to asking the whole group "who's in control?". The interesting behaviour observed with this policy is that a social protocol seemed to emerge to deal with the ambiguities. Due to the confusion (i.e. not knowing who is in control and not knowing when they will be able to obtain control), users started to pass control socially by verbally asking for control and verbally telling someone that they will be releasing control. In effect, the users were re-creating the explicit host-token policy described above. This echoes Dommel and Garcia-Luna-Aceves finding that absence of any limitation on the floor-holding time leaves mediation of competition for floors to social protocols [18]. For this application, it would seem that implicit policies (at least, the way in which we implemented it) are inappropriate. 
Using this policy, storytelling persisted; however, the story-teller was occasionally interrupted while telling the story, allowing another user would grab control of the show.

\subsubsection{Ad-hoc}

The "free-for-all" mode was implemented to determine whether users could co-ordinate themselves socially without implementing any software locks. When using this "free-for-all" mode, the result was always chaotic as all users tried to simultaneously manipulate the display. This typically included multiple users drawing on the display. Since users could draw on the display simultaneously, it was not known who drew what on the display. In one of the groups, a user requested that each user use a different colour so that they would know who drew a particular drawing. No signs of a social protocol emerging were observed. Storytelling was practically abandoned. However, due to all users interacting simultaneously, and no one having to wait for control, the users seemed to have the most fun using this mode.

\subsubsection{Conclusion}

For our evaluations, the approach of using friends and excluding one friend from the experience seemed to work out well. We generated a lot of discussion in the groups and, by having many people with material they wished to share, we generated mild conflict with users wishing to explain something more clearly than the person who currently had the floor. Even though the users were in an unnatural environment and knew that they were being observed, after using the application for a while, they seemed to become more comfortable in sharing and talking about their photos. Throughout the testing, none of the users seemed to get tired/bored from using the application. 
From these observations, there seems to be two methods of interacting with photos.

- Sharing and storytelling of photos is promoted by strict floor control policies. Not only does this ensure better behaviour from the audience, but it allows the presenter to focus on the story, knowing that their presentation will not be hi-jacked. From the observations we took of the groups, the host-token policy supported both story-led and photo-led interaction.

- Teasing friends and the 'entertainment' aspects are promoted by the ad-hoc $/$ free-for-all" mode. With the other two policies, users were willing to accommodate the policies and work with them directly (host-token) or adapt them to their needs (three-second). This was emphatically not the case with the ad-hoc mode. No attempt at structure could be maintained and the session deteriorated (or improved, depending on your view) into a form of game.

Therefore, one can conclude that applications of this nature which need to support story telling should have some form of software locking built in - our experiments would indicate that the host-token policy is the most appropriate. However, the exact form of the policy is not so important as we observed that users were more likely to pass control when verbally asked for it.

\subsection{Experiment Design 2}

\subsubsection{New Floor Control Policy}

From our previous experiment we found two methods of sharing photos supported by our prototype system. Each method is supported by a specific floor control policy. For our next experiment, we wanted to implement a single floor control policy that supports both methods of photo sharing. To achieve this, this new policy would have 
to incorporate the basic components that we believed supported these two methods of interaction.

Co-present sharing is naturally social and thus using a social protocol to pass control in this situation seems to be ideal. However, from our previous study, we found that this seems to be promoted by built in software locks. We have chosen to modify our host-token policy to promote a more social protocol as follows: The user that possesses the token will have full control of the show. The host-request interface has been removed; however, the host will be able to release the token at anytime without first having to receive a request. Using this method, other participants will have to request for the host verbally. Releasing the token will change the system to "free-for-all" mode. In this mode anyone can grab the token in a first-come-first-served basis.

Incorporating the "free-for-all" mode when the host token is released, we hope to support collaborative storytelling. In our study, we have found that participants that wanted to add narratives to the story would typically ask the host to manipulate the display when wanting to focus on particular objects in the photo. When this occurs, we expect the host would release the token, to allow these participants to collaboratively control the display while collaboratively storytelling.

This policy thus incorporates both the host-token policy and the ad-hoc policy without users having to explicitly choose the desired policy. From this we hoped to determine if these two methods of interaction can co-exist with one another. 

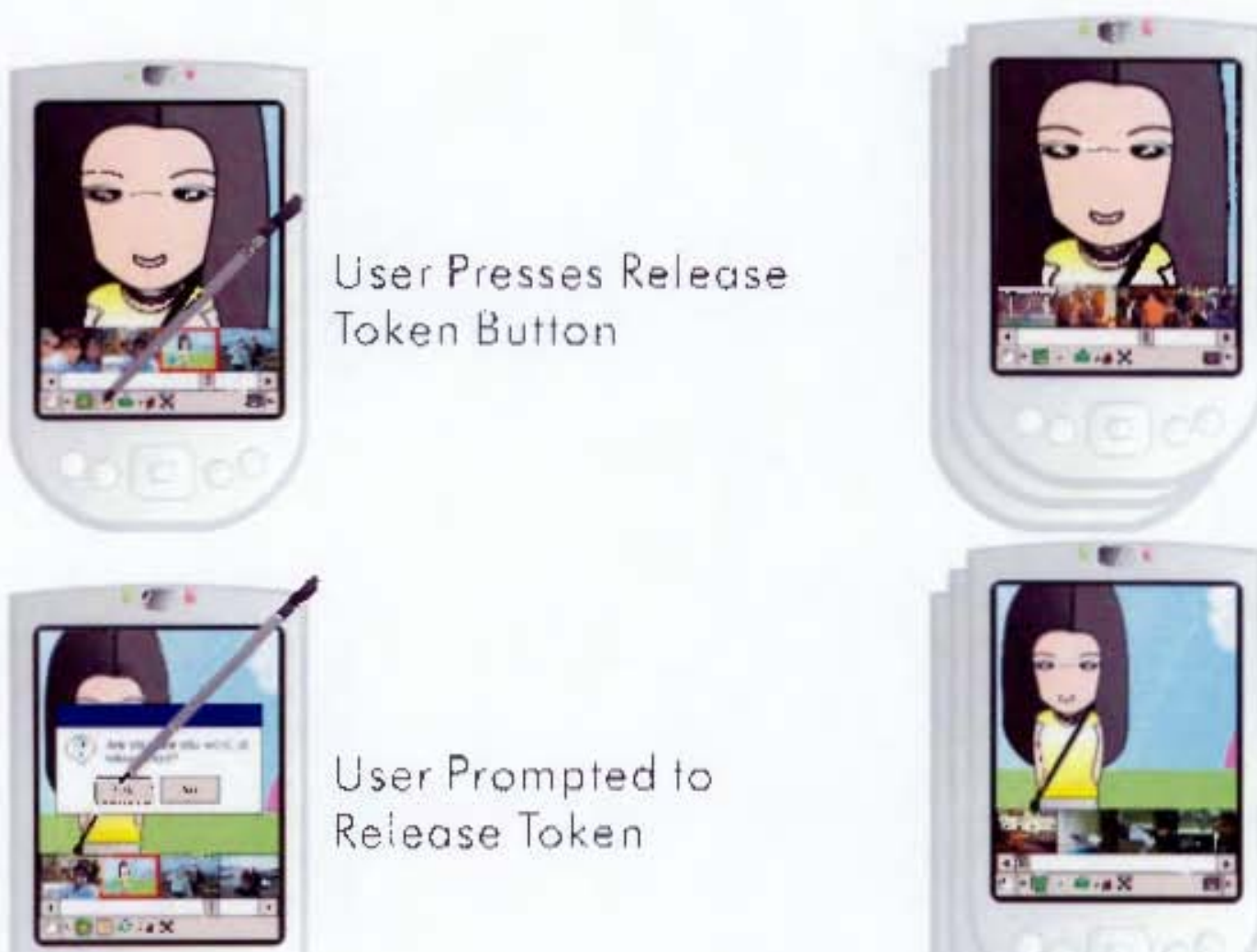

User Prompted to Release Token

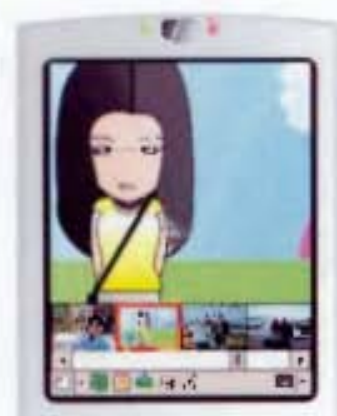

Other Users Notified of Release of Host

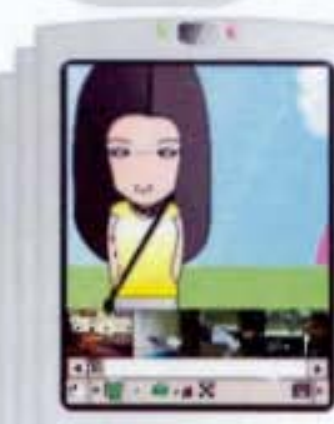




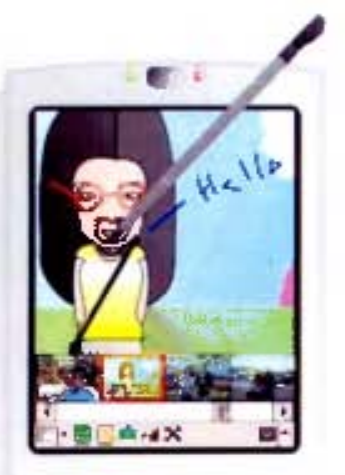

Free For All Mode When Token Released
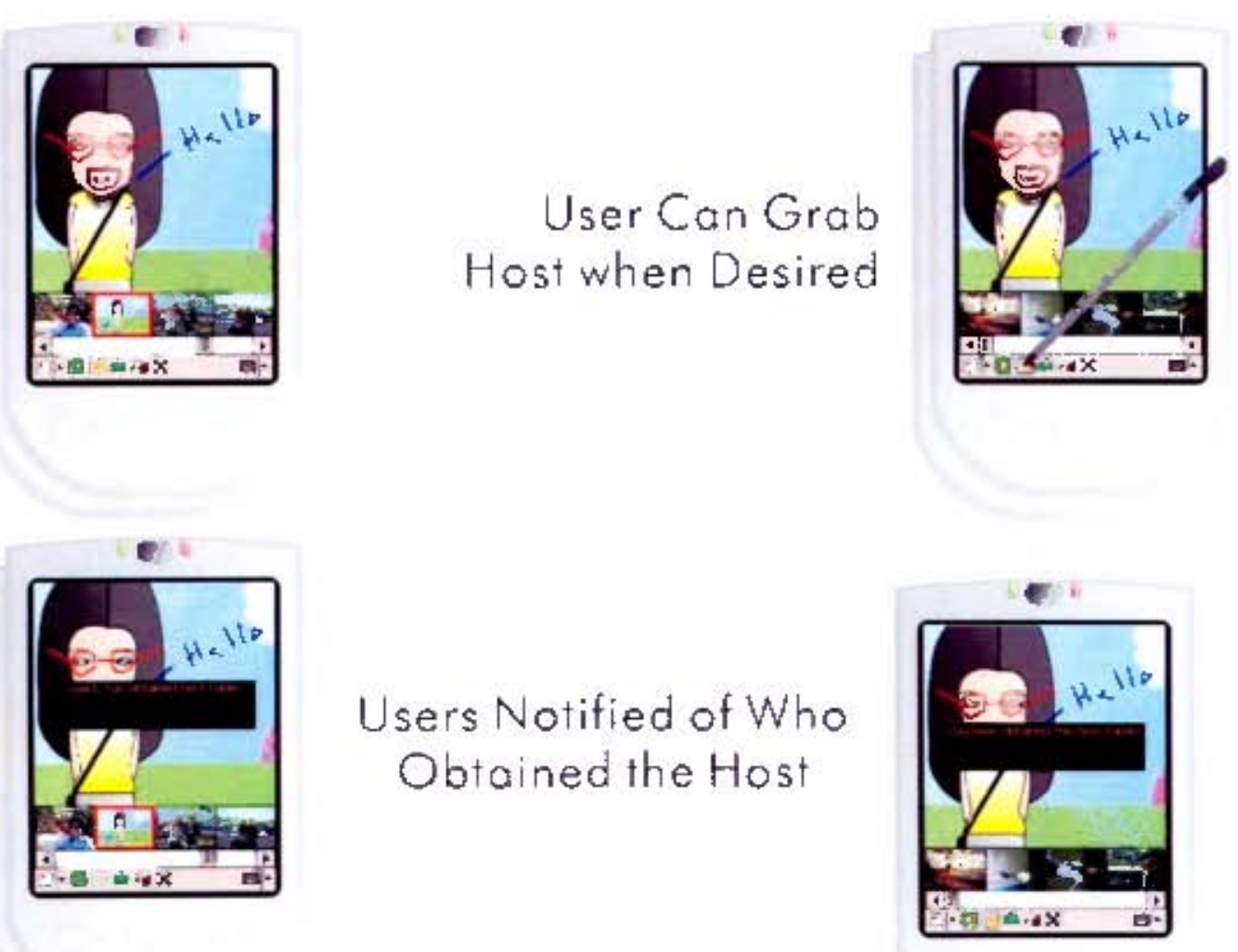

Figure 4.4, Illustration of New Policy 


\subsubsection{Experiment Design}

The objectives for our experiment are to determine

- whether this policy promotes a more social protocol.

- how users behave when having the choice of full control or "free-for-all" mode.

- how users react when the token is released without being requested by anyone.

To achieve these objectives, a naturalistic observation will be conducted as in the first experiment. Two groups were used which met the same requirements as before. To determine how users react when the token is released without first being requested, we have decided to use a variation of constructive interaction [30].

Constructive interaction can be used to determine whether the basic concepts underlying a system are well understood by users and whether its implementation, usability, and utility are satisfactory [30]. Constructive interaction typically involves pairs of users assisting each other in completing a task. The relationship between these pairs is important. Individuals can be used to engineer a situation that is guided by one person.

One of the participants from the "original capture group" will be used as a plant. This user would be briefed prior to the experiment on how to behave during the experiment. The user will be asked to release the host token when he/she feels like they have completed a story without explicitly notifying others that he/she is releasing the token. Using this constructed condition, we hoped to identify whether users realise when the token has been released. For this experiment the participants were given $\mathbf{4 5}$ minutes to talk about their photos. As with the previous experiment, a group 
discussion was held to ask the users various questions on how they felt about the application

\subsection{Experiment 2}

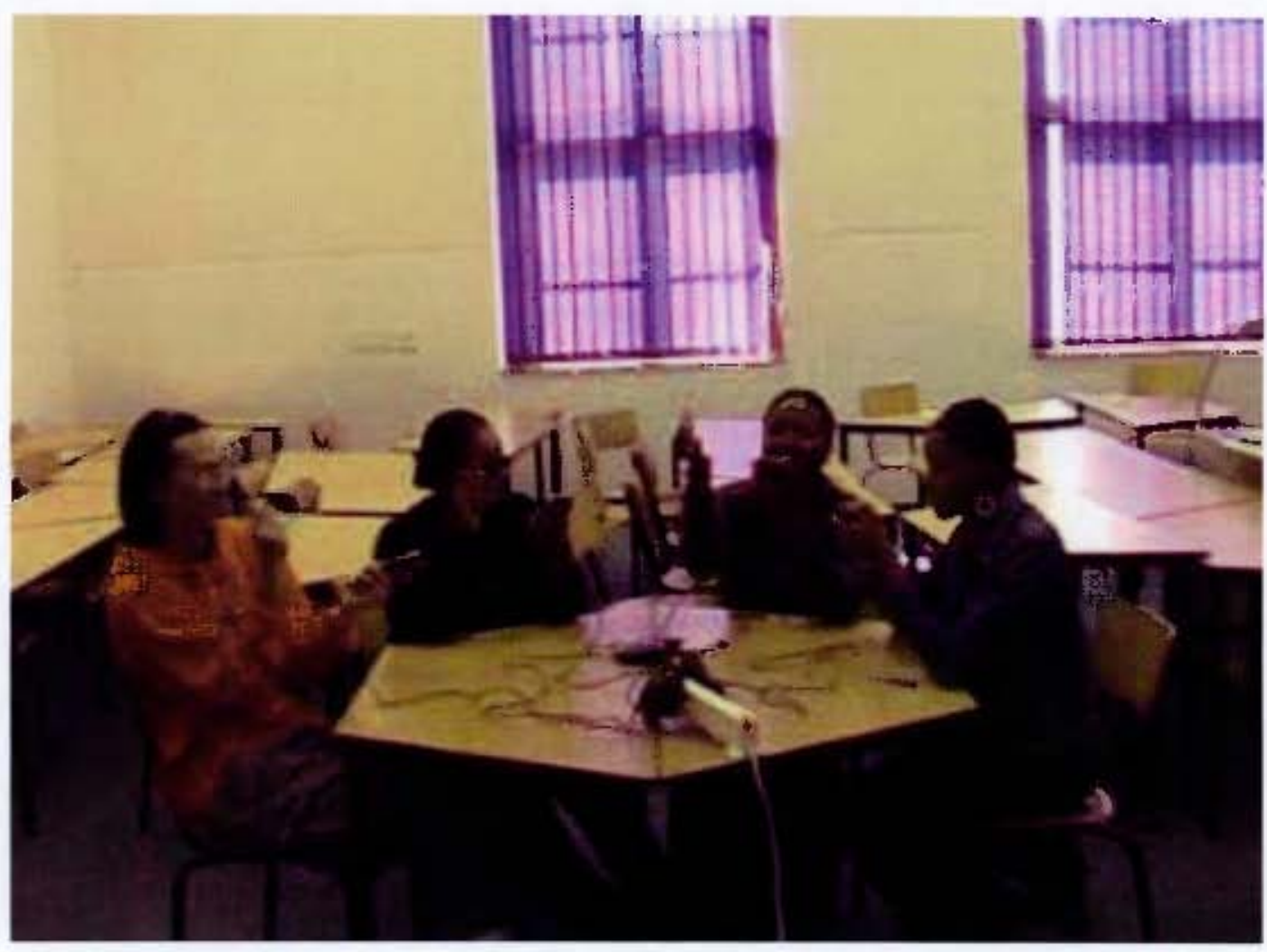

=ig̣ıre 4 E. Aे. çroup tor serond user experimen:.

\subsubsection{Group 1}

This group consisted of three females that spent their Easter holidays together. The extra friend was a male. All participants were in their early twenties and owned a camera phone.

\subsubsection{Observation}

As with our previous study, a member from the original capture group obtanned the token and began describing photos (photo-driven photo-talk). Storytelling was a collaborative effort between those of the origınal capture group who would ask the 
host to manipulate the image to comment on a part of the photo. The host decided to release the token to change the system to "free-for-all" mode so that users could manipulate the image themselves. This resulted in all participants trying to manipulate the image and push photos on the display which all participants felt was too chaotic. Due to this chaos, one of the subjects decided to grab the token to bring organisation to the group. Participants reverted to asking the host to manipulate the image again. The token was passed without explicitly asking for it. A subject would think of a photo they wanted to talk about, and ask the host to push that photo on the display. The host would then release the token so that the subject could grab the token and push the photo that he/she wanted to show themselves. The token was requested verbally when subjects had stories in mind and wanted to change the photo. This request also shows evidence of story-driven photo-talk.

When the plant released the host without explicit notification, the group did not recognise when the host was released and only realised when they noticed chaotic behaviour on the display and asked who was in control.

\subsubsection{Group Discussion}

The typical method of sharing photos in this group was via facebook, flickr and e-mail. The group found the application easy to use. They mentioned that the notification messages disappeared too quickly and that they could not read it fast enough. They did not realise when the token was released as they stopped trying to read the notification messages. All participants preferred someone owning the token as they found the "free-for-all" mode too chaotic. They suggested possibly adding a timeout mode where users would have control for a certain amount of time. They also suggested adding an interface to request the host. The group thought that this type of application could be useful in a real world situation if supported by more devices. 
Participants thought that this application was better than traditional print photos as there are seemingly "multiple" copies of the photo and you cannot zoom and draw on printed photos.

\subsubsection{Group 2}

This group consisted of 2 females and a male that attended a birthday party. The extra friend was a male.

\subsubsection{Observation}

This group found it entertaining to possess the token. They enjoyed having full control of the display. As with the previous study, photo-driven storytelling occurred initially which later led to story-driven storytelling. It was also observed that other stories were told that were not part of the photographs on hand. Storytelling was a collaborative effort between those of the original capture group who would ask the host to manipulate the image to comment on a part of the photo.

The token was passed socially when requested by other participants. Other users did not try and grab the token when the host was requested. When the token was released with out explicit notification, participants noticed this and tried to grab the token before their co-participants. When the token was released by other participants (i.e. not the plant), they explicitly notified everyone that they were releasing the token if it was not requested first. The group noticed when the host was released which excited them resulting in the entire group trying to grab the token first. The participant who obtained the token would notify the other users. Participants usually kept control until someone requested the token.

After a while, the group decided to use the "free-for-all" mode. In this mode image manipulation started out as chaotic. Everyone stopped trying to manipulate an image 
and a social protocol started to emerge. This worked for a short time, but participants reverted to having one user possess the host token.

\subsubsection{Group Discussion}

Participants typically shared photos via facebook or transfer via flash drives. Occasionally, photos were printed or shared on a television set which acted as a large group display. The group found the application easy to use. They enjoyed possessing the host and being in full control over the show. They found the "free-for-all" mode chaotic and that the system works better if someone has the host token. One user mentioned, "I think once you get over the initial jokey thing, the free-for-all mode is fine". The "jokey thing" that the user was referring to was the entertainment that users found in grabbing the host as soon as it had been released.

They disliked not being able to request the host via the interface and suggested adding that as an improvement. Compared to traditional print photos, the group thought that this method was more flexible. They liked the fact that everyone can view the same photo at the same time. They mentioned that with print photos, the photos are usually passed around and the story being told does not necessarily relate to the photo being viewed.

\subsubsection{Discussion}

With this new policy, we hoped to support both methods of photo sharing found in our previous study. The storytelling behaviours found here were the same as those of the host-token policy previously studied. Both photo-driven and story-driven storytelling were observed as well as reminiscing photo-talk. The entertainment aspect $f$ observed here was similar to that found in the three-second policy in that users enjoyed grabbing control when they could. This factor led to users not trying to 
collaborate using the "free-for-all" mode in this policy, and thus the teasing of co-participants found in our previous study, was not observed here.

In all our studies, we observed collaborative storytelling. A problem identified with our system was that, when this collaboration occurred, the other participants that added to the narrative had to ask the host to manipulate the display rather than controlling the display themselves. We wanted to support this collaboration by allowing the "free-for-all" mode when releasing the token, however this did not seem to be suffice.

The passing of the token verbally/socially was observed using this protocol. However, the groups still suggested adding an interface to request the host token. This echoes Mandryk and Inkpen's [40] study on collaborative computer games that found that players tend to interact with each other via the interface even when sitting side-by-side.

The first group had difficulty identifying when the token had been released and only identified this by chaotic behaviour on the display. This could be a result in most users ignoring the notification message which is only displayed for three seconds. The second group however had no problems reading the notification message, and thus could identify when the token was released.

\subsubsection{Conclusion}

From our observations, our new policy seems to be an improvement over our host-token policy. Users could release the token when they desired and were not irritated by multiple interface requests. This policy did cause users to pass control socially, however it seems that users prefer to interact with each through the interface and wanted an interface to request the host. If this were to be implemented again, a possible solution to multiple interface requests is to set a time limit between requests. 
With this new policy, the entertainment factor found in our ad-hoc policy was not observed here. Users found this "free-for-all" mode chaotic and unpredictable. It seems that the entertainment factor (drawing and teasing) found with this policy only emerges when this policy is enforced and the subjects have no other software lock options.

The token being released seems to be identified mainly by the notification message that is displayed on the screen for a few seconds. If this message is ignored by the user, they do not realise that the token has been released. 


\section{Conclusion}

Our research was aimed at improving the social interaction of sharing digital photographs by recreating the compelling experiences found in traditional photo sharing. The basic elements that we identified to achieve this were mobility, spontaneity and face-to-face interaction. From the literature surveyed, face-to-face interaction previously has only been supported by SDG (Single Display Groupware). We found this limiting as large displays were not portable and thus spontaneity can not occur and mobile devices that were used as the shared display were limiting in that only 1 or 2 people can view the screen at a time.

To tackle these limitations, we developed a prototype that was mobile, and supported a large group of participants being able to view and interact with the shared photos synchronously. This was achieved by allowing each participant to use their own device as their own personal display of the shared photos. The interaction was supported by various floor control policies which determined when a user could manipulate the shared display.

\subsection{Research Questions}

To answer our research questions, two experiments were conducted. These were in the form of naturalistic observations to observe and understand users' behaviours with our system.

Would people really be interested in seeing someone else's photos on the device - or will the screen simply be too small to be engaging?

From our observations, users seemed to enjoy themselves interacting with each other and talking about their photos using our prototype. Many stories were told including stories that were not related to the photographs available. From our post-experiment 
discussions with the users, they mentioned that this method of sharing photographs has many advantages over traditional printed photographs. These advantages include the ability to zoom, draw, and show all the photos in the collection - when printing photos, only a selected set of photos are printed due to the cost of printing. Another advantage noted by the users when using our prototype is that the stories being told are the same as the photos being viewed. With traditional print photos, the stories being told are not always about the photos in the hand of a given participant as photos get passed around while stories are being told.

From our first experiment, we identified a method of photo sharing interaction supported by our prototype which is unrelated to storytelling. This was in the form of entertainment. Participants engaged in teasing each other by drawing on the photographs. This resulted in all participants interacting simultaneously which seemed to be highly entertaining for the users.

How should the system manage the social interaction - should anyone be allowed to broadcast an image at any time, or does control need to be moderated through some convention such as token passing?

Our first experiment was used to evaluate three types of floor control policies to determine which best supports photo-sharing using our prototype. The three policies included an explicit, implicit and a "free-for-all" policy.

The explicit policy, whereby the user that possesses the token holds control, seemed to work best for storytelling. This policy allowed the host to constantly know when he/she was in control over the show without worrying about others "stealing" control. However, as only one user could be in control at a time, when collaborative storytelling emerged, the other participants that wanted to add narration had to ask the host to manipulate the display on their behalf. One problem that all groups 
identified was that the host could not be released without being requested yet. This meant that a user could not release the token when he/she did not want control any longer.

The implicit policy allowed users to keep control of the display as long as they performed a control action within three seconds of their last control action. Thereafter, control would be automatically released and assigned to the next person who performed a control action. Using this policy, the participants were both confused and frustrated as they constantly did not know who was in control and constantly being denied control as someone else would grab control before them. From all this confusion and frustration, a social protocol emerged to coordinate control in more orderly fashion. This policy also did not seem to support collaborative storytelling as users continued asking the current user in control to manipulate the display on their behalf.

To determine whether users could socially coordinate themselves without any software locks, a "free-for-all" mode was included. In this mode, all users could interact with the display simultaneously. This mode led to storytelling among participants to decrease and teasing and drawing on the photographs to increase. Even though storytelling ceased, the participants seemed to have the most fun sharing photos and socially interacting this way

From the results of our first experiment we found that our system does support compelling collaboration amongst the groups. The policies implemented seemed to give us a good idea in which software locks are needed to best support photo sharing. We found two methods of photo-sharing that took place, storytelling and entertainment. An explicit policy seems to work best for storytelling and a "free-for-all" policy seems to work best for entertainment purposes. 
With our first experiment, none of our policies really seemed to support collaborative storytelling. Our second prototype aimed to support both storytelling and entertainment photo-sharing found in our first prototype as well as support collaborative storytelling. To do this, we implemented a single policy whereby the user that possess the host token controls the show. The host-request interface was removed and the host-release interface was added for the host to release the token when desired. When no user holds the token the system changes to free-for-all mode whereby any user can control the display. In this mode, any user can also "grab" the token giving them full control of the display. Implementing this policy, we envisioned supporting the storytelling found in our previous host-token policy when a single user possesses the token. When the token is released, we anticipated both collaborative storytelling and entertainment photo-sharing. Removing the host-request interface, we also anticipated users passing control socially as from our previous experiment we found that users were more likely to release control when asked verbally.

Observations from our second experiment showed that users preferred to be in full control over the show and disliked uncoordinated collaboration when in "free-for-all" mode. They found "free-for-all" mode too chaotic and preferred a more stable environment.

For our experiments we attempted to create a more relevant social setting between participants as our prototype was designed for co-located collaboration. From our first experiment, we found that users were more likely to pass control to another user from a verbal request even though requests were triggered via the interface. In our second experiment, requests for control could only be achieved verbally. From our observations this seemed to work as expected, however, from the post group discussion, the participants suggested adding a request interface as an improvement 
in the application. It seems that users prefer to interact with each other via the interface even when interacting face-to-face with each other. A possible reason for this could be that when users request control verbally and the host releases control, the requester is not guaranteed control. In our observations, the requester always obtained control when the host released control, however, assurance of obtaining this control might be preferred.

\subsection{Contributions}

Our research describes both traditional and current photo sharing techniques. We identified the social interaction of traditional photo-sharing and found that this interaction is not supported by current available systems. We have developed a photo sharing prototype that uses WYSIWIS (What-You-See-Is-What-I-See) on mobile devices to support co-present photo-sharing. From the literature surveyed, this seems not to have been pursued before. Our research provides an insight to an alternative to shared displays focusing on photo-sharing and describes interaction ideas that could be used for other mobile CSCW applications.

\subsection{Future Work}

The entertainment factor observed in our first experiment was not observed in our second experiment. It is possible that this behaviour only emerges when users are forced to use the "free-for-all" mode. This mode was not used for a long period of time by the participants in our second experiment and therefore the "teasing" behaviour did not have time to emerge.

Throughout the experiment, participants had enough photos to talk about throughout the evaluation. It is possible that since there was always a photo to talk about, the motivation of storytelling was overpowering the creative behaviour of "teasing". 
Increasing the experiment time or requesting the participants to bring less photos could give different results.

If users were all from the original capture group, this could decrease storytelling and increase reminiscing talk which could increase the motivation for "teasing" as all members would be in the photographs.

The groups used in our evaluations were limited to four participants. The policies implemented seem to work well with this group size. Using a larger group of participants might result in different behaviours emerging.

Our prototype was evaluated in a controlled setting. Different uses/behaviours could also emerge if users had time to use this prototype as part of their everyday lives.

For future work, all these factors can be tested by modifying our experiment slightly. Other future work could include adding more features to the prototype. Media sharing could be included where audio and video could be broadcast in addition to photos. 


\section{References}

[1] Apted, T., Kay, J., Quigley, A., Tabletop Sharing of Digital Photographs for the Elderly, Conference on Human Factors in Computing Systems, Proceedings of the SIGCHI conference on Human Factors in computing systems

[2] ACDSee. http://uww.acdsystems.com

[3] Adobe Photoshop Album. http://www.adobe.com

[4] Balabanović, M., Chu, L.L., Wolff, G.J. Storytelling with Digital Photographs, Proceedings of the SIGCHI conference on Human factors in computing systems

[5] Ballagas, R., Yu, K., Hibino, S. Taking and Sharing Pictures with Phonecams: An Ethnographic Study. Technical report, Stanford University, 2004

[6] Bassoli, A., Moore, J., and Agamanolis, S., tunA: Socialising Music Sharing on the Move (book chapter), in Kenton O'Hara and Barry Brown (eds), Consuming Music Together: Social and Collaborative Aspects of Music Consumption Technologies, Springer, 2006.

[7] Begole, J., Rosson, M.B., Shaffer, C.A., Flexible Collaboration Transparency: Supporting Worker Independence in Replicated Application-Sharing Systems, ACM Transactions on Computer-Human Interaction (TOCHI), Volume 6, Issue 2 (June 1999), pp 95-132

[8] Berry, L., Bartram, L., Booth, K.S., Role-Based Control of Shared Application Views, Symposium on User Interface Software and Technology, Proceedings of the 18th annual ACM symposium on User Interface Software and Technology, pp. 23-32 
[9] Boyd, J. Floor Control Policies in Multi-User Applications, Conference on Human Factors in Computing Systems, INTERACT '93 and $\mathrm{CHI}$ '93 conference companion on Human factors in computer Systems, pp. 107-108

[10] Brinck, T., Gomez, L.M. A Collaborative Medium for the Support of Conversational Props, Computer Supported Cooperative Work, Proceedings of the 1992 ACM conference on Computer-Supported Cooperative Work. pp. 171-178

[11] Chalfen, Richard (1987): Snapshot Versions of Life. Bowling Green, Ohio: Bowling Green State University. Popular Press.

[12] CORPORATE Maypole Project Team, Maypole Highlights: What Makes Kids Tick?, Interactions, Volume 6, Issue 6, pp. 80-83

[13] Counts, S., Fellheimer, E. Supporting Social Presence through Lightweight Photo Sharing On and Off the Desktop. ACM Press (2004)

[14] Crabtree, A. Rodden, T. Mariani, J. Collaborating around collections: informing the continued development of photoware. Proceedings of the 2004 ACM Conference on Computer Supported Cooperative Work, pp. 396-405

[15] Crowley, T., Milazzo, P., Baker, E., Forsdick, H., and Tomlinson, R. 1990. MMConf: An infractructure for building shared multimedia applications. In Proceedings of the ACM Conference on Computer-Supported Cooperative Work (CSCW '90, Los Angeles, CA, Oct. 7-10), F. Halasz, Ed. ACM Press, New York, NY, 329-342.

[16] CU-SeeME http://uww.cuworld.com 
[17] Deering, S.E., Cheriton, D.R. Multicast Routing in Datagram Internetworks and Extended LANs, Symposium Proceedings in Commpunications Architectures and Protocols, 1988, pp. 55-64

[18] Dommel, H.P., Garcia-Luna-Aceves, J.J., Floor Control for Multimedia conferencing and Collaboration, Multimedia Systems, Volume 5, Issue 1, 1997, pp. 23-38.

[19] Elrod, S., Bruce, R., Gold, R., Goldberg, D., Halasz, F., Janssen, W., Lee, D., McCall, K., Pedersen, E., Pier, K., Tang, J., Welch, B. Liveboard: A Large Interactive Display Supporting Group Meetings, Presentations, and Remote Collaboration, Proceedings of the SIGCHI conference on Human Factors in Computing Systems. pp. 599-607

[20] Erramilli, A., Singh, R.P. A Reliable and Efficient Multicast for Broadband Broadcast Networks, Proceedings of the ACM workshop on Frontiers in Computer Communications Technology, 1987, pp. 343-352

[21] Flickr.com. http://uww.flickr.com

[22] Floyd, S., Jacobson, B, Liu, C.G., McCanne, S., Zhang, L., A Reliable Multicast Framework for Light-Weight Sessions and Application Level Framing. IEEE/ACM Transactions on Networking (TON), Volume 5, issue 6, Dec 1997, pp.784-803

[23] Fouss, J.D., Chang, K.H. Classifying Groupware, Proceedings of the 38th annual on Southeast regional conference, pp127-124

[24] Frohlich, D, Kuchinsky, A., Pering, C., Don, A. and Ariss, S. Requirements for Photoware. Hewlett Packard Laboratories. 
[25] Giller, V., Tscheligi, M., Sefelin, R., Mäkelä, A., Puskala, A., Karvonen, K. Maypole Highlights: Image Makers, Interactions, Volume 6, Issue 6, pp 12-15

[26] Greenberg, S. Sharing Views and Interactions with Single-User Applications, Conference on Supporting Group Work, Proceedings of the ACM SIGOIS and IEEE TC-OA conference aon Office Information Systems, 1990, pp 227-237

[27] Greenberg, S., Marwood, D. Real Time Groupware as a Distributed System: Concurrency Control and its Effect on the Interface, Computer Supported Cooperative Work, Proceedings of the 1994 ACM Conference on Computer Supported Cooperative Work, 00.207-217

[28] iPhoto. http://www.apple.com/iphoto

[29] Jacobsson, M., Rost, M., Håkansson, M., Holmquist, L.E. Push!Music: Intelligent Music Sharing on Mobile Devices. Adjunct Proceedings of UbiComp 2005, Tokyo, Japan

[30] Kahler, H., Kensing, F., Muller, M., Methods \& Tools: Constructive Interaction and Collaborative Work: Introducing a Method for Testing Collaborative Systems, Interactions, Volume 7, Issue 3, 2000, pp. 27-34

[31] Kindberg, T, Spasojevic, M., Fleck, R., Sellen, A. How and Why People Use Camera Phones. Consumer Applications and Systems Laboratory, HP Laboratories Bristol, 2004.

[32] Kirk, D. Sellen, A., Carsten, R. Wood, K. Understanding Photowork, Conference on Human Factors in Computing Systems, Proceedings of the SIGCHI conference oh Human Factors in computing systems.

[33] Koskinen, I., Kurvinen, E., Lehtonen, TK. Mobile Image. IT Press 2002 
[34] Kurvinen, E. (2003): Only When Miss Universe Snatches Me: Teasing in MMS Messaging. Proceedings of the International Conference on Designing Pleasurable Products and Interfaces (DPPI 03). pp. 98-102

[35] Jones, M., Marsden, G. “Mobile Interaction Design”. John Wiley \& Sons, Ltd, 2006

[36] Li, D., Muntz, R. COCA: Collaborative Objects Coordination Architecture, Proceedings of the 1998 ACM conference on Computer Supported Cooperative Work, pp.179-188

[37] Li, D., Wang, Z., Muntz, R.R. "Got COCA?" A New Perspective in Building Electronic Meeting Systems, Proceedings of the International Join Conference on Work Activities Coordination and Collaboration, 1999, pp. 89-98

[38] Liechti, O., Ichikawa, T., A Digital Photography Framework Enabling Affective Awareness in Home Communication, Personal Technologies (Springer Verlag), Volume 4, Issue 1, 2000, pp 6-24

[39] Mäkelă, A., Giller, V., Tscheligi, M., and Sefelin, R. (2000): Joking, Storytelling, Artsharing, Expressing Affection: A Field Trial of How Children and their Social Network Communicate with Digital Images in Leisure Time. Proceedings of the Conference on Human Factors in Computing Systems. pp. 548-555.

[40] Mandryk, R.L., Inkpen, K.M., Physiological Indicators for the evaluation of Co-Located Collaborative Play, Proceedings of the 2004 ACM Conference on Computer Supported Cooperative Work, pp. 102-111

[41] Microsoft Live Messenger http://get.live.com/messenger/overview

[42] Microsoft Netmeeting http://www.microsoft.com/windows/netmeeting/ 
[43] Miller, A.D., Edwards, W.K. Give and Take: A Study of Consumer Photo-Sharing Culture and Practice, Proceedings of the ACM Conference on Human Factors in Computing Systems (CHI 2007)

[44] Myers, B.A., Mobile Devices for Control, Lecture Notes in Computer Science; Vol. 2411, Proceedings of the $4^{\text {th }}$ International Symposium on Mobile Human-Computer Interaction

[45] Myers, B.A., Stiel, H., Garguilo, R., Collaboration using multiple PDAs connected to a PC, Proceedings of the 1998 ACM conference on Computer supported cooperative work

[46] Ngadi, M.A., Doherty, B.S. Multi-Users Shared Whiteboard Under Web-Multicast System, Proceedings of the 1st Internation Symposium on Information and Communication Technologies

[47] O'Hare, N., Gurrin, C., Lee, H., Murphy, N., Smeaton, A.F., Jones, G.J.F. Video Demonstrations and Visions: My Digital Photos: Where and When?, Proceedings of the 13th Annual ACM Internation Conference on Multimedia MULTIMEDIA '05

[48] Smith, R.B., Hixon, R, Horan, B. Supporting Flexible Roles in a Shared Space, Computer Supported Cooperative Work, Proceedings of hte 1998 ACM conference on Computer Supported Cooperative Work, pp. 197-206

[49] Picassa Software. http://www. picassa.net

[50] Pictavision http://uww.pictavision.com

[51] Preece, J., Rogers, Y., Sharp, H., Interaction Design: Beyond Human-Computer Interaction, 2002, John Wiley \& Son's 
[52] Pullen, J. Reliable Multicast Network Transport for Distributed Virtual Simulation, Proceedings of the 3rd International Workshop on Distributed Interactive Simulation and Real-Time Applications. 1999. pp.59

[53] Rekimoto, J. A multiple device approach for supporting whiteboard-based interactions, Proceedings of the SIGCHI conference on Human Factors in computing Systems. 1998, pp. 344-351

[54] Rost, M., Jacobsson, M. and Holmquist, L.E., Push!Photo:Informal Photo Sharing in Ad-Hoc Networks, In Extended abstracts of UbiComp 2006, Orange County, USA

[55] Salovaara, A., Jacucci, G., Oulasvirta, A., Saari, T., Kanerva, P., Kurvinen, E., Tiitta, S. (2006). Collective creation and sense-making of mobile media. Proceedings of $\mathrm{CHI}$ 2006, ACM Press, New York, pp. 1211-1220

[56] Salvucci, D.D., Markley, D., Zuber, M., Brumby, D.P., Task \& Attention: iPod Distraction: Effects of Portable Music-Player Use on Driver Performance, Proceedings of the SIGCHI conference on Human Factors in Computing Systems $\mathrm{CHI}$ ' 07

[57] Sanneblad, J., Holmquist, L.E., Designing collaborative games on handheld computers, International Conference on Computer Graphics and Interactive Techniques, ACM SIGGRAPH 2003 Sketches \& Applications

[58] Sanneblad, J., Holmquist, L.E., OpenTrek: A Platform for Developing Interactive Networked Games on Mobile Devices, Proceedings of Mobile $\mathrm{HCl} 2003$, Fifth International Symposium on Human Computer Interaction with Mobile Devices and Services 
[59] Sarvas, R., Oulasvirta, A., Jacucci, G. Building Social Discourse Around Mobile Photos - A Systemic Perspective MobileHCl 2005, Salzburg, Austria

[60] Schmidt, A., Lauff, M. and Beigl, M. Handheld CSCW. Workshop on Handheld CSCW at CSCW' '98, 14 November, Seattle1998.

[61] Shen, C., Lesh, N., Vernier, F., Personal Digital Historian: Story Sharing Around the Table, Interactions, Volume 10, Issue 2, Winds of Change, pp. 15-22

[62] Van House, N. A., Davis, M., Takhteyev, Y., Ames, M., Finn, M. (2004). The Social Uses of Personal Photography: Methods for Projecting Future Imaging Applications

[63] Vernier, F. Lesh, N.B., Shen, C, Visualization Techniques for Circular Tabletop Interfaces, ACM Advanced Visual Interfaces (AVI), May 2002

[64] Vronay, D. Farnham, S. Davis, J. PhotoStory: Preserving Emotion in Digital Photo Sharing, Virtual Worlds Group, Microsoft Research 


\section{Appendix 1}

Left screenshot will represent devicc $A$, and Right screenshot device $B$.

\section{Startup Screen:}

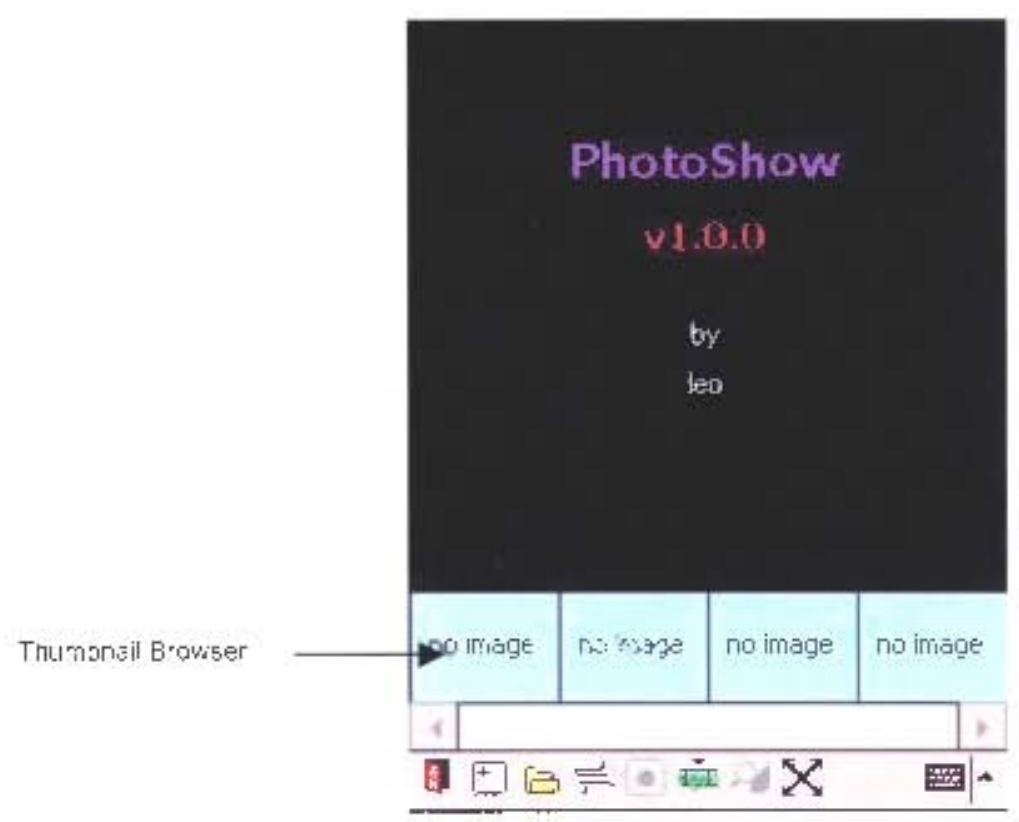

Toolbar Buttons (from left to right):

- Exit: exits application

- Options: change your user name and multicast group

- Photo Folder: select the folder with the photos that you want to share

- Connect/Disconnect: grean if connected. Yellow dot on top if you have the host token.

- Requcst Host: used to request host token

- Hide/Show Thumbnail Browser

- Photo Tools: activates tools to zoom, draw, rotate, etc.

- Full screen: changes to full screen mode 


\section{Connecting:}

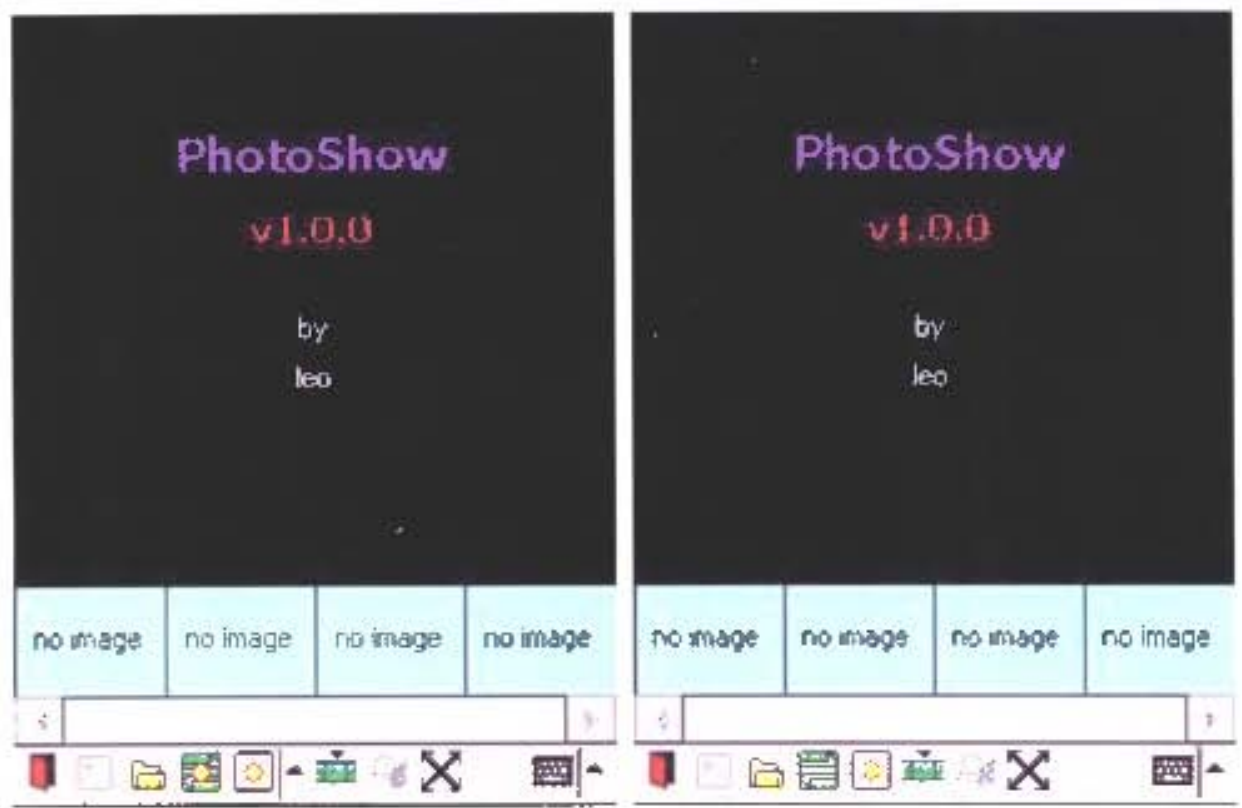

When connect button is pressed, the first user to connect will obtain the host token.

This is identified by the yellow dot on the connect button.

\section{Loading Thumbnails:}

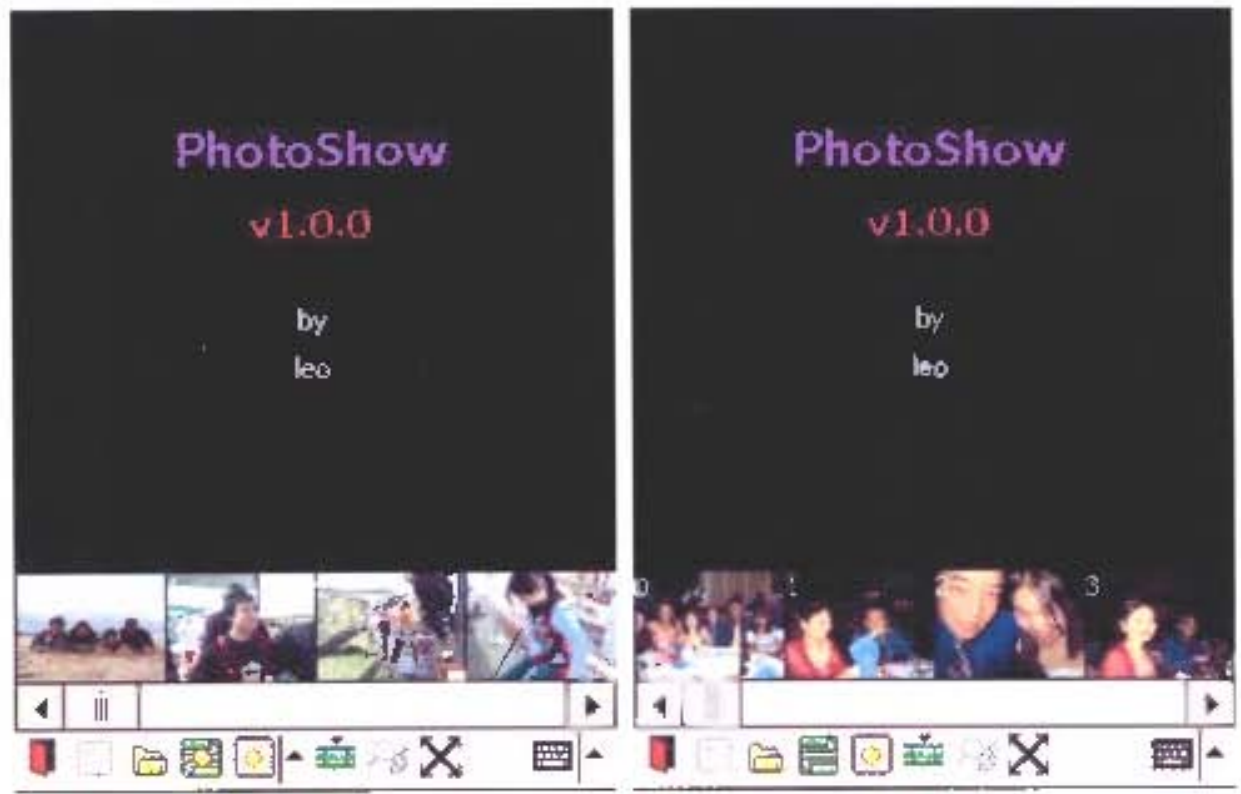

Selecting a photo folder loads thumbnails in the thumbnail browser. 


\section{Selecting a photo:}
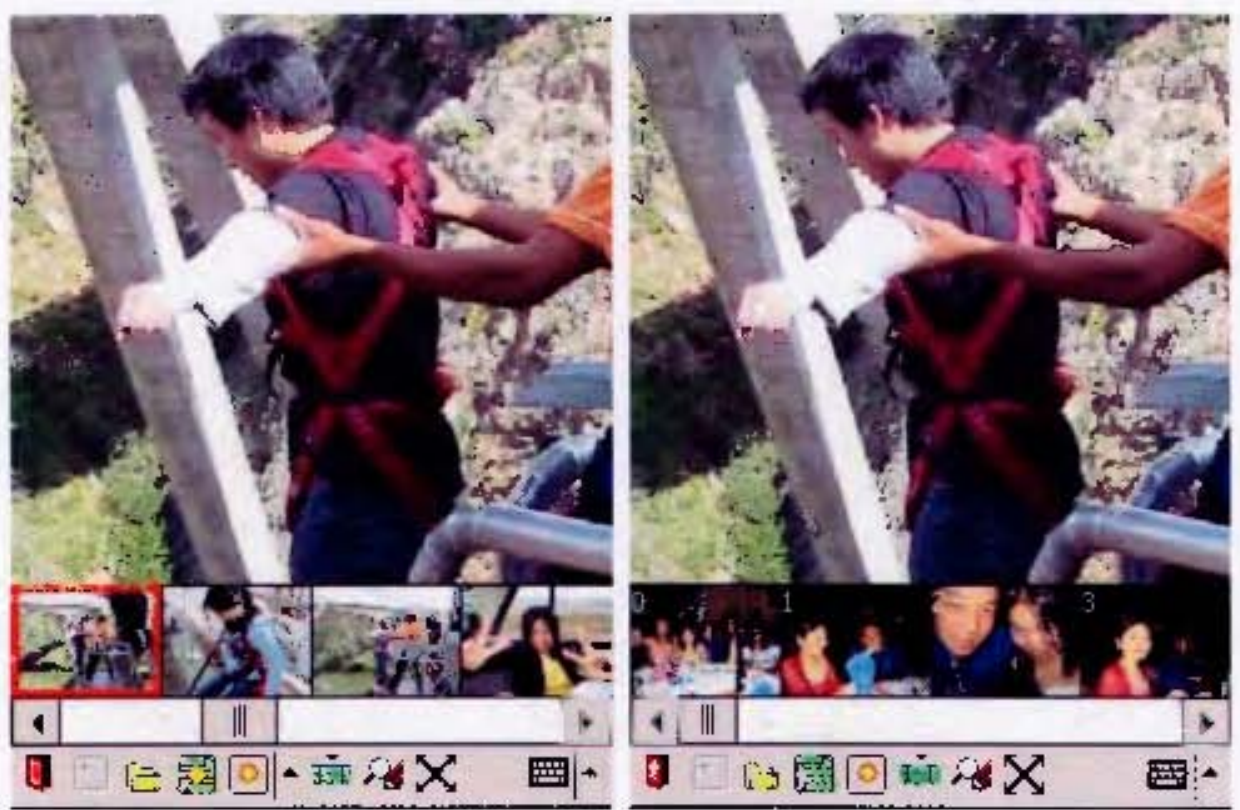

Selecting a photo will send the photo to all other devices and display it on their screen.

\section{Panning:}
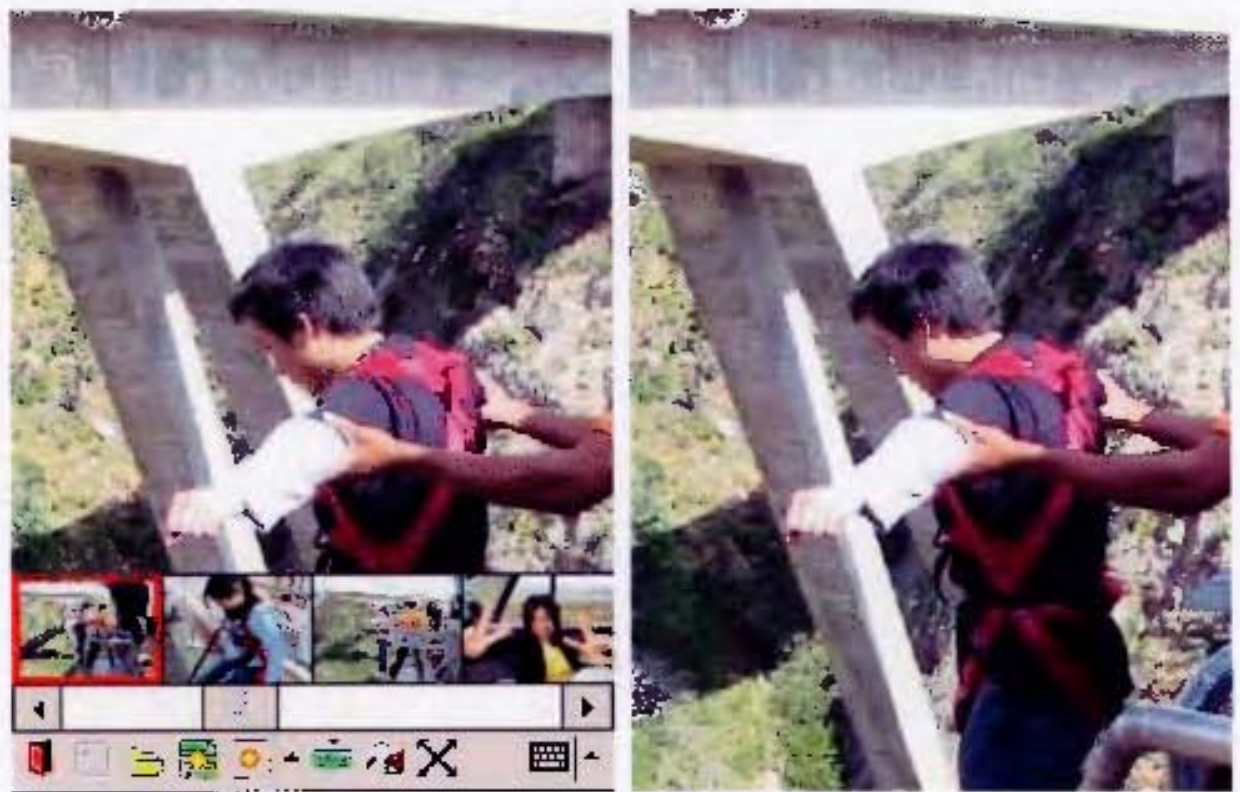

Pan the image my just dragging the image with the stylus. Device B is in full screen mode. 


\section{Photo Tools:}

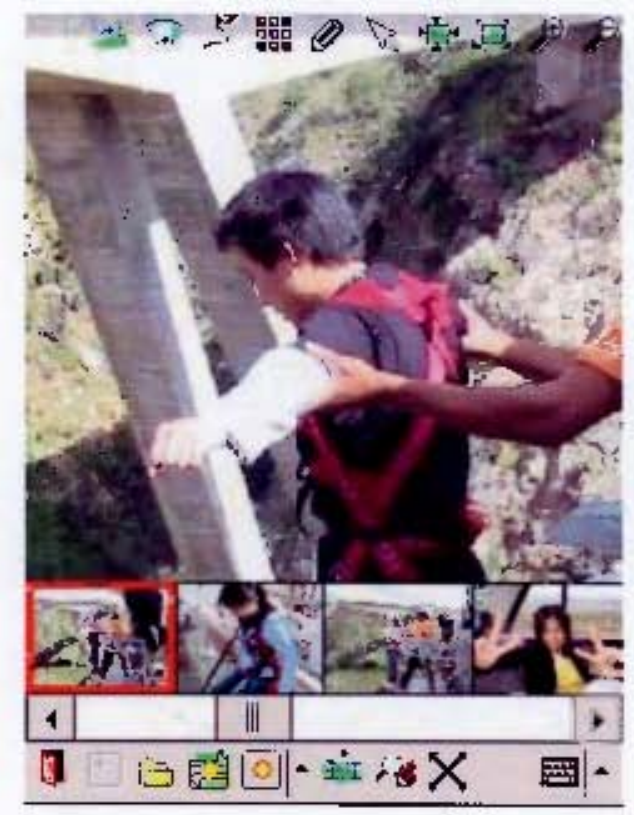

Photo Toos (from left to right):

- Rotate: Rotates image 90 degrees.

- Clear: Clears drawings

- Line Width: Select Line width for drawing

- Penn Colour: Select Colour of Pen

- Draw: Activate/Deactivate Pen

- Pointer: used to point at things

- Best Fit: zooms image to fit screen

- Actual Size: zooms image to actual size of image

- Zoom In

- Zoom Out 


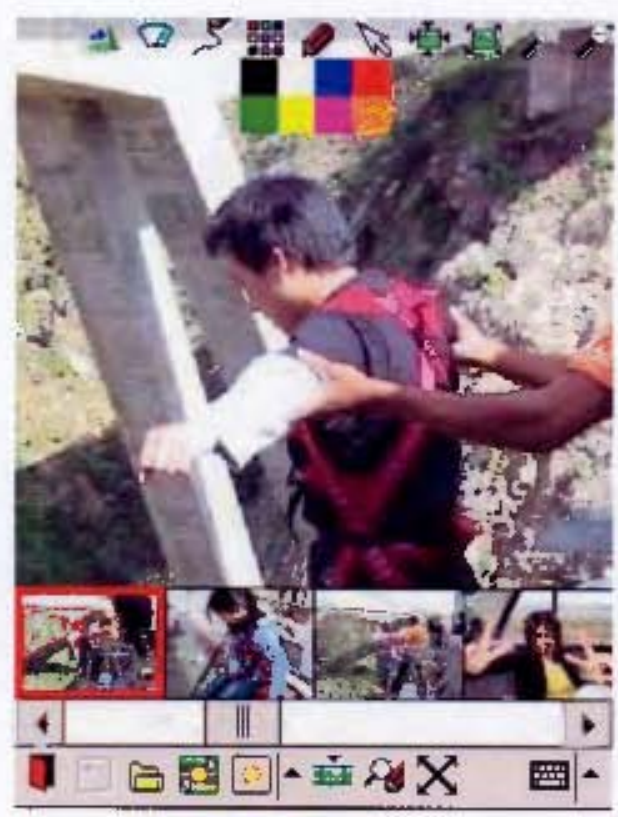

A simple colour selector for drawing was implemented.

\section{Pointer:}
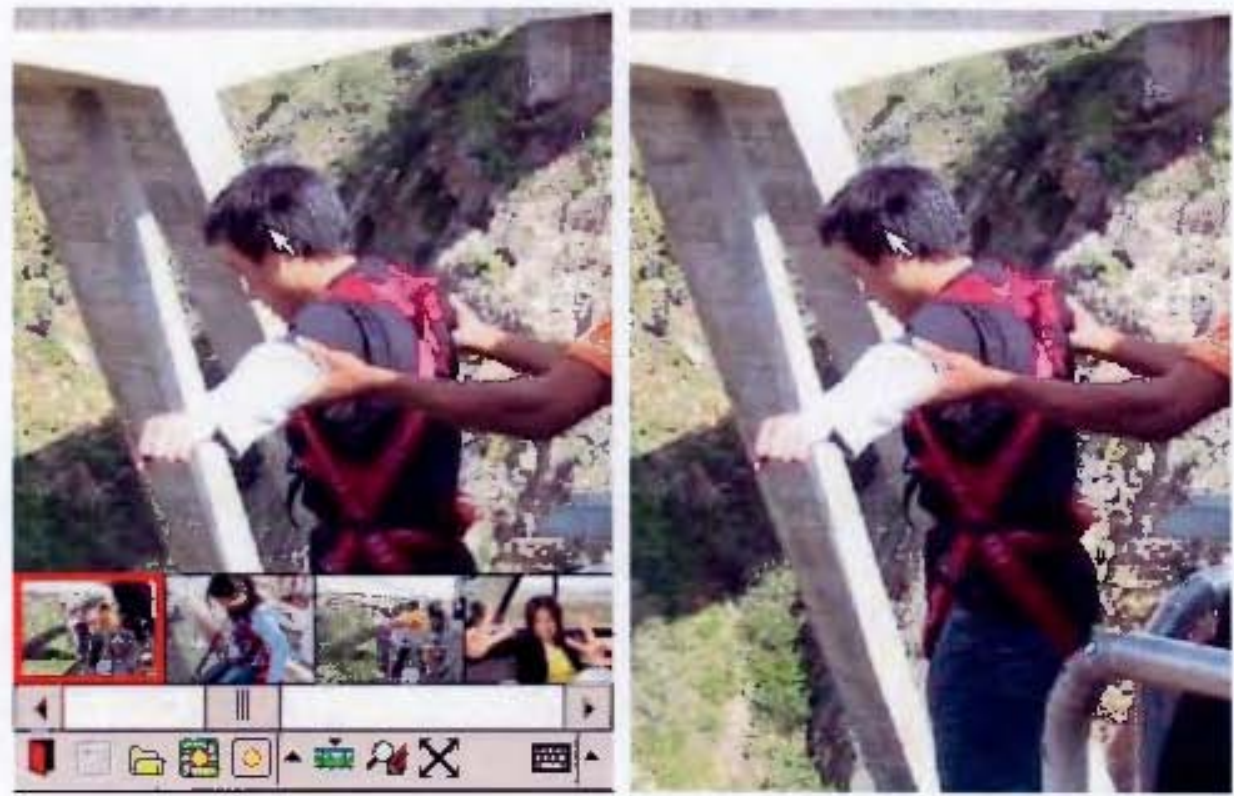

Pointer can be used to point at things in the photo. 


\section{Drawing, Thumbnail Navigator:}
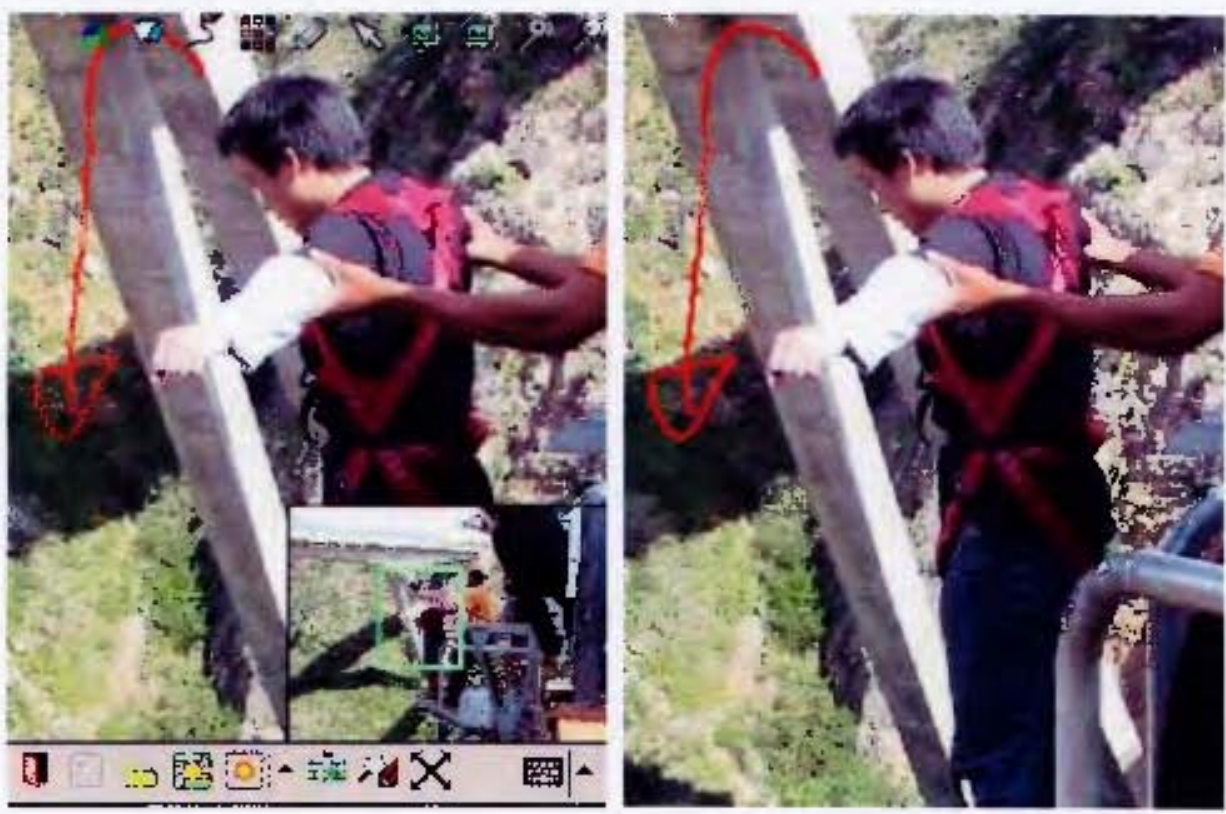

A simple drawing. The thumbnail navigator on the Device A can be uscd to pan

images. It is displayed for 3 seconds if you tap the screen

\section{Rotation, Coordination Control Selection:}
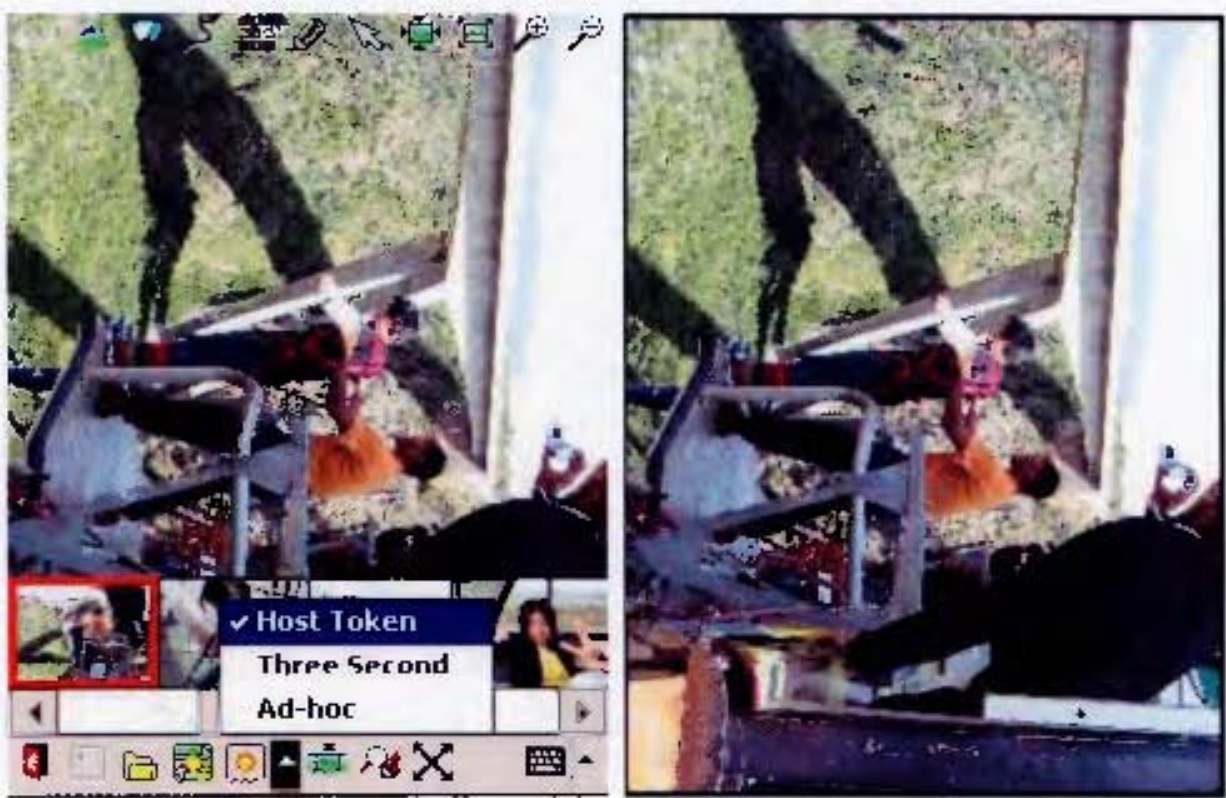

Simple Rotation. Menu to select floor control policy. Floor control policy can be changed by the user that possesses the host token. 
Ad-hoc, Three Second Control:

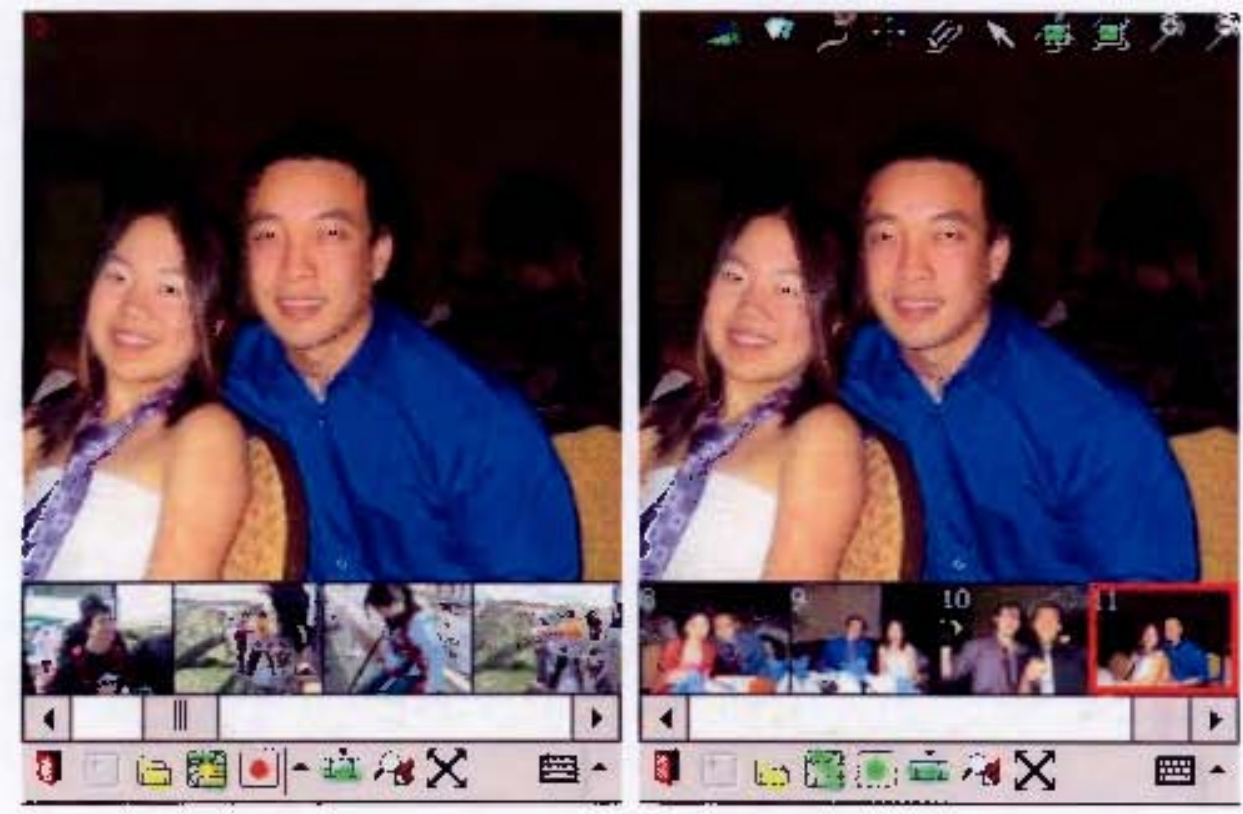

I hree-Second Policy is selected. Device B has selected a photo and it is displaycd on all other devices. On Device $\mathrm{A}_{\text {r }}$ the red circle on the top left corner represents that this device cannot peiform a control action. When the circle disappears, the device will be allowed to perform control action. The red and green dots on the toolbars also represent the control status information. 


\section{Appendix 2}

\section{Video Release/Confidentiality agreement}

Participants name:

E-mail/Phone:

I have been provided with information about the procedure for the Co-Present Mobile Photo Sharing study and I am happy to take part.

The handling of my data for this study has been explained to me. No notes or logs will bear any information by which I might be identified. In addition, unless I agree to the Special Release below, any video or audio collected during the usability study will be viewed by the experimenter.

I understand that I can with withdraw from the Co-Present Mobile Photo Sharing study at any point without prejudice or penalty of any kind.

$\square$ I would / $\square$ would not be happy for the Department of Computer Science to use small excerpts of video from my session for educational purposes. No excerpts would be shown that could be construed as unflattering or embarrassing for me.

Signature:

Date:

Experimenter's Name:

Date:

Experimenter's Signature: 